\title{
Construction, assembly and tests of the ATLAS electromagnetic end-cap calorimeters
}

ATLAS Electromagnetic Liquid Argon Endcap Calorimeter Group
M. Aleksa, ${ }^{a}$ F. Astesan, ${ }^{c}$
D. Banfi, ${ }^{f}$ F. Barreiro, ${ }^{d}$ P. Barrillon, ${ }^{f, 1}$
C. Benchouk, ${ }^{f, 2}$
W. Bertoli, ${ }^{c}$ J. Bremer, ${ }^{a}$ H.M. Braun, ${ }^{k}$ B. Canton, ${ }^{c}$ L. Carminati, ${ }^{f}$ T. Carli, ${ }^{a}$ C. Cerna, ${ }^{e}$
M. Chalifour ${ }^{j}$ J.L. Chevalley, ${ }^{a}$ M. Citterio,${ }^{f}$ J. Collot, ${ }^{b}$ G. Costa, ${ }^{f}$ P. Dargent, ${ }^{e}$
B. Dekhissi, ${ }^{h}$ J. E. Derkaoui, ${ }^{h}$ F. Djama, ${ }^{e}$ C. Fabre,,${ }^{a}$ A. Fallou, ${ }^{i}$ M. Fanti, ${ }^{f}$
P. Fassnacht, ${ }^{a}$ D. Fournier, ${ }^{i}$ C. Gabaldon Ruiz, ${ }^{d}$ F. Gianotti, ${ }^{a}$ J. Giner, ${ }^{e}$ A. Gonidec, ${ }^{a}$
F. Henry-Couannier, ${ }^{e}$ L. Hervas,${ }^{a}$ T. Hott, ${ }^{a, 3}$ F. Hubaut,,${ }^{e}$ D. Imbault, ${ }^{c}$ P. Karst,${ }^{e}$

V.A. Kazanin, ${ }^{g}$ K.Y. Kotov, ${ }^{g}$ L. Labarga, ${ }^{d}$ D. Lacour, ${ }^{c}$ B. Laforge, ${ }^{c}$ P. Lopez-Iturriaga, ${ }^{d}$ G. Macé,$^{i}$ V. Malychev,${ }^{g}$ L. Mandelli, ${ }^{f}$ P.S. Mangeard,${ }^{e}$ B. Mansoulié, ${ }^{j}$ C.P. Marin, ${ }^{a}$

A.L. Maslennikov, ${ }^{g}$ M. Mazzanti, ${ }^{f}$ J.P Meyer, ${ }^{j}$ E. Monnier, ${ }^{e}$ E. Nebot Del Busto, ${ }^{d}$ V. Niess, ${ }^{e}{ }^{e}$ C. Oliver, ${ }^{d}$ I.A. Orlov, ${ }^{g}$ P.M. Pailler, ${ }^{a}$ S.V. Peleganchuk, ${ }^{g}$ J. Del Peso, ${ }^{d}$

A. Pleskatch, ${ }^{a}$ P. Pralavorio, ${ }^{e}$ S. Prat,${ }^{i}$ P. Puzo,${ }^{i}$ M. Raymond,,${ }^{e} a$ B. Resende, ${ }^{e}$

S. Rodier ${ }^{d}{ }^{d}$. Romero, ${ }^{d}$ F. Rossel, ${ }^{c}$ D. Sauvage, ${ }^{e, 8}$ A.G. Schamov, ${ }^{g}$ Ph. Schwemling, ${ }^{c}$ J. Schwindling, ${ }^{j}$ C. Serfon,,$e$ J. Setien, ${ }^{e}$ L. Serin, ${ }^{i}$ A. Soukharev, ${ }^{g}$ C. De La Taille, ${ }^{i}$

A. Talyshev, ${ }^{g}$ G. Tartarelli, ${ }^{f}$ Y.A. Tikhonov, ${ }^{g}$ S. Tisserant, ${ }^{e *}$ J. Toth,,${ }^{e, 6}$ G. Unal, ${ }^{a}$

G. Vandoni, ${ }^{a}$ J.J. Veillet, ${ }^{i}$ V. Vuillemin, ${ }^{a}$ H.G. Wilkens ${ }^{a}$ and H. Zhang ${ }^{e, 7}$

${ }^{a}$ CERN, CH - 1211 Geneva 23, Switzerland, Switzerland

${ }^{b}$ Laboratoire de Physique Subatomique et de Cosmologie,

CNRS-IN2P3, Université Joseph Fourier,

INPG, 53 avenue des Martyrs, FR - 38026 Grenoble Cedex, France

${ }^{c}$ Laboratoire de Physique Nucléaire et de Hautes Energies,

Université Pierre et Marie Curie (Paris 6), Université Denis Diderot (Paris-7),

IN2P3-CNRS, Tour 33, 4 place Jussieu, FR - 75252 Paris Cedex 05, France

${ }^{d}$ Departamento de Fisica Teorica, Facultad de Ciencias, Universidad Autonoma de Madrid,

ES - 28049 Madrid, Spain

${ }^{e}$ CPPM, Aix-Marseille Université, CNRS/IN2P3, Marseille, France

${ }^{f}$ INFN Milano and Università di Milano, Dipartimento di Fisica,

via Celoria 16, IT - 20133 Milano, Italy

${ }^{g}$ Budker Institute of Nuclear Physics (BINP), RU - Novosibirsk 630 090, Russia 
${ }^{h}$ Laboratoire de Physique Théorique et de physique des Particules, Université Mohammed

Premier, Oujda, Morocco

${ }^{i} L A L$, Univ. Paris-Sud, IN2P3/CNRS, Orsay, France

${ }^{j}$ CEA, DSM/DAPNIA, Centre d'Etudes de Saclay, FR - 91191 Gif-sur-Yvette, France

${ }^{k}$ University of Wuppertal, Wuppertal, Germany

${ }^{1}$ Now at LAL, Univ. Paris-Sud, IN2P3/CNRS, Orsay, France

${ }^{2}$ Now at Faculté de Physique, Université des Sciences et de la Technologie Houari Boumediène, BP 32 El-Alia 16111 Bab Ezzouar, Alger, Algérie

${ }^{3}$ Now at DESY, Hamburg and Zeuthen, Notkestr. 85, D-22603 Hamburg, Germany

${ }^{4}$ Now at Laboratoire de Physique Corpusculaire, IN2P3-CNRS, Universite Blaise Pascal, FR - 63177 Aubiere Cedex, France

${ }^{5}$ Now at Fakultät für Physik der Ludwig-Maximilians-Universität München,

Am Coulombwall 1, DE - 85748 Garching, Germany

${ }^{6}$ Currently at KFKI, Budapest, Hungary, Supported by the MAE (France), the HNCFTD

(Contract F15-00) and the Hungarian OTKA (Contract T037350)

${ }^{7}$ Also at Institute of HEP, Chinese Academy of Sciences, P.O. Box 918, CN-100049 Beijing

${ }^{8}$ Deceased

E-mail: tisserantecppm.in2p3.fr

ABSTRACT: The construction and the assembly of the two end-caps of the ATLAS liquid argon electromagnetic calorimeter as well as their test and qualification programs are described. The work described here started at the beginning of 2001 and lasted for approximately three years. The results of the qualification tests performed before installation in the LHC ATLAS pit are given. The detectors are now installed in the ATLAS cavern, full of liquid argon and being commissioned. The complete detectors coverage is powered with high voltage and readout.

KEYWORDS: Calorimeters, Large detector systems for particle and astroparticle physics, Liquid detectors, Cryogenic detectors.

${ }^{*}$ Corresponding author 


\section{Contents}

1. Introduction 2

2. General description 1

3. Mechanical components of a module 9

3.1 Presampler 9

3.2 Absorbers 10

3.2.1 Lead production $\quad 13$

3.2.2 Stainless steel sheets 13

3.2.3 Longitudinal bars 13

3.2.4 Absorber fabrication 14

3.3 Mechanical structure 16

3.3.1 General description 16

3.3.2 Outer rings 18

3.3.3 Intermediate and inner rings 18

3.3.4 Indexing rings 19

3.3.5 Production 19

3.4 Electrodes 19

3.5 Spacers 21

4. Module assembly 23

5. Cold electronics and cabling 24

5.1 Summing boards and mother-boards 25

5.2 Signal and calibration cables 27

5.3 High voltage boards and cables 27

5.4 Cabling procedure 28

5.5 Monitoring instruments 28

6. Cold and beam tests 28

6.1 Experimental set-up 29

6.2 High voltage tests 29

6.3 Signal and calibration test 29

6.4 Beam test results 31

7. End-cap cryostat 32

7.1 Cryostat characteristics

7.2 Feedthroughs characteristics 34 
8. Integration 34

8.1 EMEC wheel assembly 34

8.2 High voltage tests during wheel assembly 37

8.3 Electromagnetic calorimeter insertion into the cryostat 37

8.4 Electrical tests during integration 41

8.4 .1 High voltage tests 41

8.4 .2 Signal and calibration test

8.4.3 Capacitance measurement 41

9. Qualification tests of the calorimeter 42

9.1 Cooling down 42

9.2 High voltage tests 43

9.3 Electrical tests 43

10. Conclusions 45

\section{Introduction}

Experimentation in particle physics will reach its next high energy frontier at the Large Hadron Collider (LHC). At the LHC two proton beams of $7 \mathrm{TeV}$ energy will collide every $25 \mathrm{~ns}$ at four points of its perimeter. The multipurpose ATLAS detector, has been built to operate in one of those interaction points. Main focus of the LHC physics program is the study of the electroweak symmetry breaking where the relevant cross sections are predicted to be well below the picobarn level.

Calorimeters will play a crucial role at the LHC. In contrast to other detectors, their intrinsic resolution improves with energy, which makes them very suitable detectors at high energy machines. At LHC, calorimeters will be the leading detectors in many measurements for the reconstruction of physics channels of prime interest. Many physics processes, whose discovery or detailed study are expected, will manifest themselves through final states with electrons or photons. Therefore precise measurement of the characteristics of the electrons and photons will be of upmost importance. Moreover, the calorimeters will also be used for triggering and for real time filtering of interesting events.

This can be illustrated quantitatively through a few physics channels. The discovery of the Higgs boson will rely on complementary production and decay processes. Among them, the $H \rightarrow \gamma \gamma$ channel will contribute for a Higgs mass range of $110-140 \mathrm{GeV} / \mathrm{c}^{2}$, while $H \rightarrow$ eeee will be essential if the Higgs mass is larger than $130 \mathrm{GeV} / \mathrm{c}^{2}$. Monte Carlo studies have shown that to separate them from the background a mass resolution of $1 \%$ is needed [1, 2]. To achieve it, for both channels, the sampling term must be kept at the level of $\sim 10 \% / \sqrt{E(G e V)}$ or below, while the constant term should be smaller than $1 \%$. The electromagnetic energy scale must be known to $0.1 \%$ to minimize the systematic error contribution to the mass measurement. These channels require also a linearity of the response better than $0.5 \%$ up to $130 \mathrm{GeV}$. The photon direction must 
be measured with high accuracy, so that the angular contribution to the width of the reconstructed $\gamma \gamma$ invariant mass stays lower than the energy reconstruction contribution. This means an angular resolution of $\sim 50 \mathrm{mrad} / \sqrt{E(\mathrm{GeV})}$ on the polar angle. An excellent photon/jet separation is needed in order to suppress $\gamma$-jet and jet-jet backgrounds to the $H \rightarrow \gamma \gamma$ channel. In particular the electromagnetic calorimeter must be able to reject isolated high- $p_{T} \pi^{0}$. It must also be able to separate the two photons from a $\pi^{0}$ decay up to transverse energies of $60 \mathrm{GeV}$ and to measure the energy fraction carried by each of them.

An excellent energy resolution with a small constant term is needed not only for Higgs physics, but also, for example, for Z' or ' '' searches. In addition a channel such as $Z^{\prime} \rightarrow e^{+} e^{-}$requires to measure electrons with energies up to a few $\mathrm{TeV}$. The dynamic range of the cell readout electronics must cover from $30 \mathrm{MeV}$ (noise level) up to $\sim 1 \mathrm{TeV}$.

To meet the above requirements on electromagnetic calorimetry, ATLAS has chosen the liquid argon technique because of its intrinsic linear behavior, stability of the response in time and radiation tolerance. The geometry has been chosen of accordion type because it allows very good hermeticity, since the high voltage and signal cables run only on the front and back faces of the detector. As a byproduct, this geometry increases the absorber rigidity which improve their relative positioning and hence contributes to the reponse uniformity. In addition, the accordion geometry minimizes inductances in the signal paths, allowing the use of the fast shaping needed to cope with the $25 \mathrm{~ns}$ interval between bunch collisions at LHC. The first studies of liquid argon calorimetry with the accordion geometry date back to 1992 [3, 4]. The R\&D collaboration RD-3 built several accordion-based prototypes to evaluate their performances for a LHC experiment. Among them, and in order to profit from the same technology development, an attempt was made to adapt the accordion concept to the end-cap region. It was successfully tested in particle beams [5] demonstrating that this concept could also be used for this region. After the choice of liquid argon technology by the ATLAS collaboration for its electromagnetic calorimetry [1, 6, 可, the geometry of the detectors and the design of their components were improved and rethought in view of a series production, using the experience gained with the RD-3 prototypes.

In this paper we describe the construction, assembly and tests of the end-cap part of the ATLAS electromagnetic calorimeter (hereafter called EMEC for Electro-Magnetic End-Cap). The barrel or central part (hereafter called EMB) is described in [8].

The EMEC assembly line was validated by the construction (in 1999) and extensive testing of a last prototype, called Module 0 [9], using final or close to final components for its construction. The fabrication of some of the elements of the calorimeter started in the beginning of 2000, and the construction of the detectors took place from 2001 to mid-2004. During this period three modules were calibrated with particle beams. The first end-cap was assembled at CERN, then inserted into its cryostat in 2003, while the second one was completed in 2004. The two end-caps have been lowered in the experiment pit in December 2005 and April 2006 respectively.

The paper is organized as follows. In section 2 , a general description of the electromagnetic end-cap calorimeter is given. The section 3 deals with the fabrication of the various mechanical components necessary to assemble an EMEC module. The module assembly is explained in section 4 . The cold electronics and the detector cabling are described in section 5. The cold and beam tests performed to validate the module construction are explained in section 6 . The end-cap cryostat design is summarized in section 7 . Section 8 describes the assembly of eight modules as a 


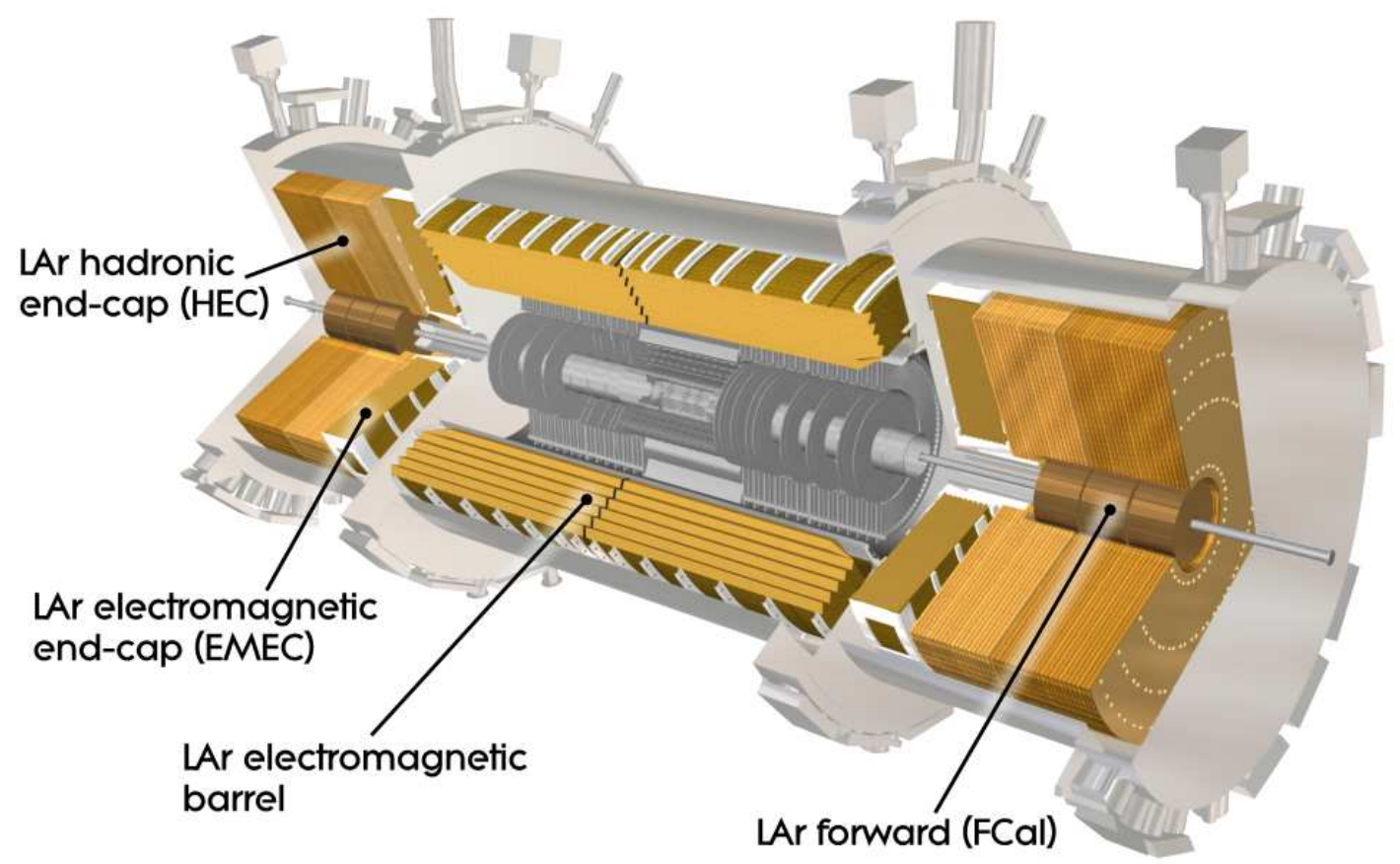

Figure 1. Cut-away view of the ATLAS liquid argon calorimeters.

wheel, then the insertion of a wheel into its cryostat. The qualification tests of the complete wheels performed at warm and at cold are the subject of section 9. Finally, the last section is dedicated to conclusions.

\section{General description}

The ATLAS electromagnetic end-cap calorimeters consist of two wheels, one on each side of the electromagnetic barrel calorimeter [8]. Figure 1] shows the liquid argon ATLAS calorimetry with the two end-caps clearly visible. One of the real EMEC wheels is shown inside the cryostat in figure 2

The EMEC is a lead-liquid argon sampling calorimeter with interleaved accordion-shaped absorbers and electrodes. Each wheel, $630 \mathrm{~mm}$ thick with external and internal radii at ambient temperature of $2098 \mathrm{~mm}$ and $330 \mathrm{~mm}$ respectively, weighs 27 tons. It covers the pseudorapidity range $1.375<|\eta|<3.2$. Due to the barrel cryostat and the inner detector services the matter in front of the EMEC, in the pseudorapidity range $1.5<|\eta|<1.8$, would degrade the energy measurement. In order to recover good performances in this region, a liquid argon presampler is implemented in front of the end-cap calorimeter. 


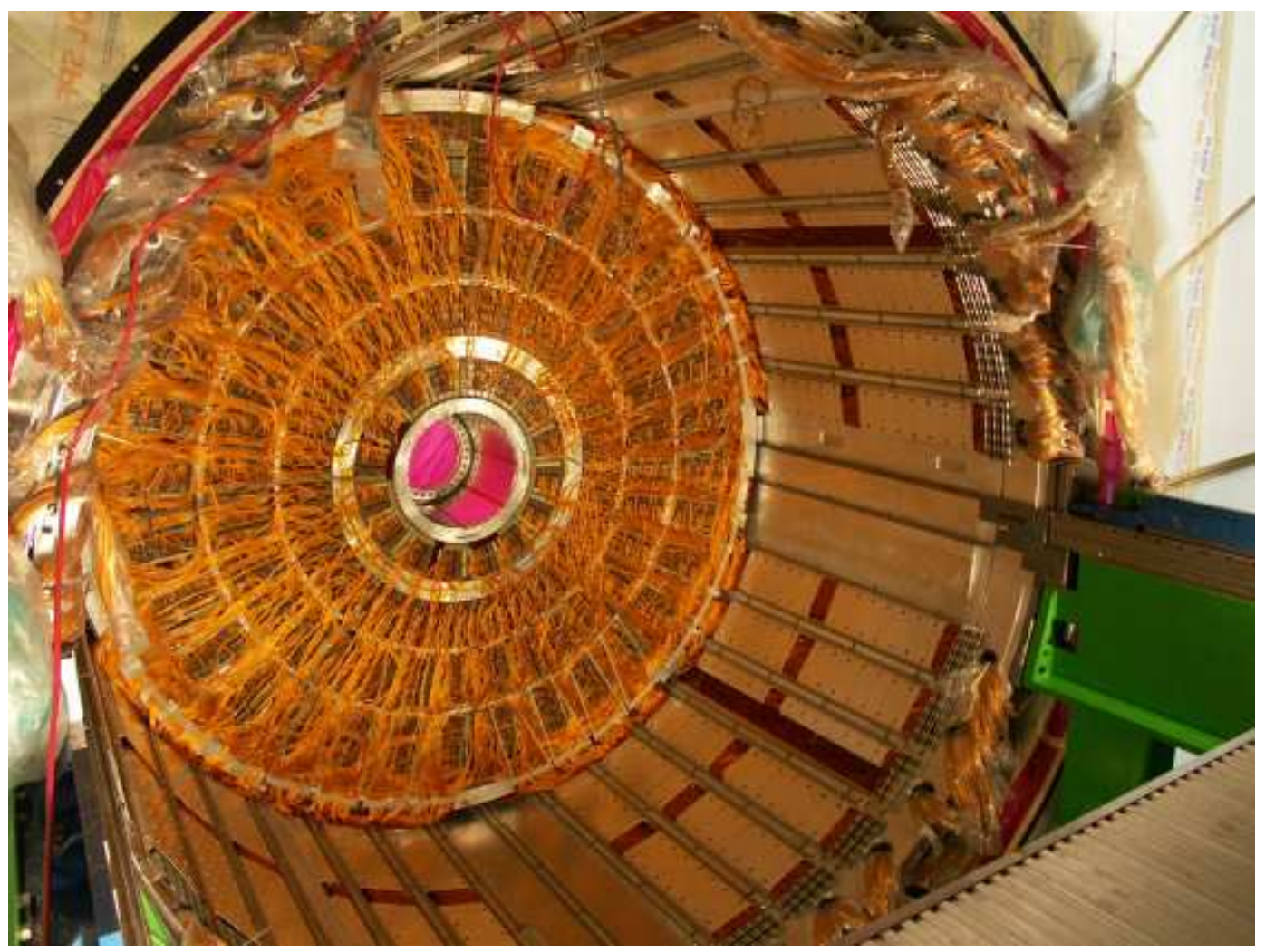

Figure 2. An electromagnetic end-cap calorimeter inside its cryostat. Mother-boards and cables on the back side are visible as well as the cable trays mounted on the cold wall of the cryostat.

To accommodate the accordion geometry in the end-cap region, the absorber plates are arranged radially like the spokes of a bicycle wheel. The accordion waves are parallel to the front and back edges of the detector and run in depth (parallel to $z$-axis), as shown in figure 3. To ensure a good azimuthal uniformity of the response, the combined thickness of liquid argon and absorber crossed by the particles must be independent of the azimuth angle $(\phi)$. As the liquid argon gap increases with the radius, it is necessary to adapt the wave height and the folding angle at each radius. For technical reasons the folding angle must be kept between about $60^{\circ}$ and $120^{\circ}$. It was impossible to fulfill this requirement in covering the full pseudorapidity range with only one accordion structure. Thus each end-cap calorimeter consists of two coaxial wheels. The boundary between these two wheels is located at $|\eta|=2.5$, it is projective and it matches the boundary of the rapidity range covered by the ATLAS charged particle tracking system $|\eta|<2.5$. The corresponding gap is $3 \mathrm{~mm}$ wide and mainly filled with low density material. Further in this paper, these two parts will be referred as outer and inner wheels.

For logistics at construction and easiness of handling, each end-cap wheel is divided into eight wedge-shaped modules without introducing any discontinuity along the azimuthal angle (thanks to the accordion concept). A photograph of a module is shown in figure $\theta$.

Each end-cap is made of 768 absorbers interleaved with readout electrodes in the outer wheel and 256 in the inner wheel. The absorbers are made of lead plates cladded with two layers of $0.20 \mathrm{~mm}$ thick stainless steel to ensure absorber rigidity and smooth surface for high voltage. The 


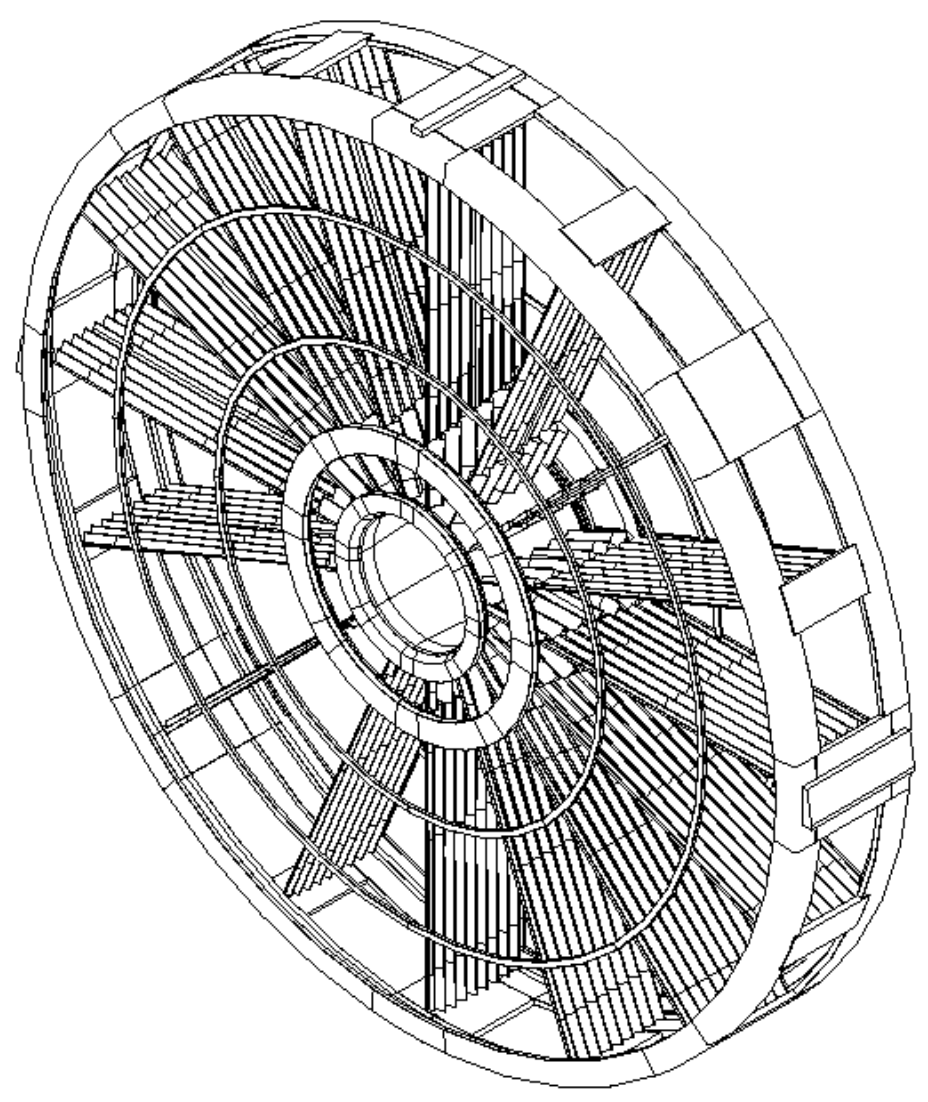

Figure 3. Schematic view of an electromagnetic end-cap wheel, with only a few absorbers to show their radial arrangement. Some elements of the structure described in section 3.3 (rings, strechers, feet and top key) are also visible.

absorbers are held and positioned to the calorimeter structure by two glass-epoxy bars which are glued longitudinally along the two straight edges of the absorbers, corresponding to its front and back sides. The lead plates are $1.7 \mathrm{~mm}$ thick in the outer wheel and $2.2 \mathrm{~mm}$ in the inner wheel. The total active thickness of an end-cap calorimeter is larger than $24 X_{0}$, except for $|\eta|<1.475$. It increases from 24 to $38 X_{0}$ when $|\eta|$ runs from 1.475 to 2.5 , and from 26 to $36 X_{0}$ for $2.5<|\eta|<3.2$.

A readout electrode is a flexible large size printed board [10]. It consists of three conductive layers (copper) insulated by polyimide sheets (kapton). The two outer layers hold a high voltage potential to drift the ionization charge created by charged particles in the liquid argon gaps. In order to minimize the risk to lose a full liquid argon gap in case of high voltage failure, the two external layers are supplied by two different power supplies. The signal is collected by capacitive coupling on the inner layer. The three copper layers are etched to define the $\eta$ granularity and the longitudinal segmentation. In the outer wheel, signals from the different pads are readout at two sides of the electrode, i.e. at the front and back of the calorimeter. In the inner wheel signals are all read out at the back side only, because of the higher radiation level, and also because there are only two longitudinal compartments. 


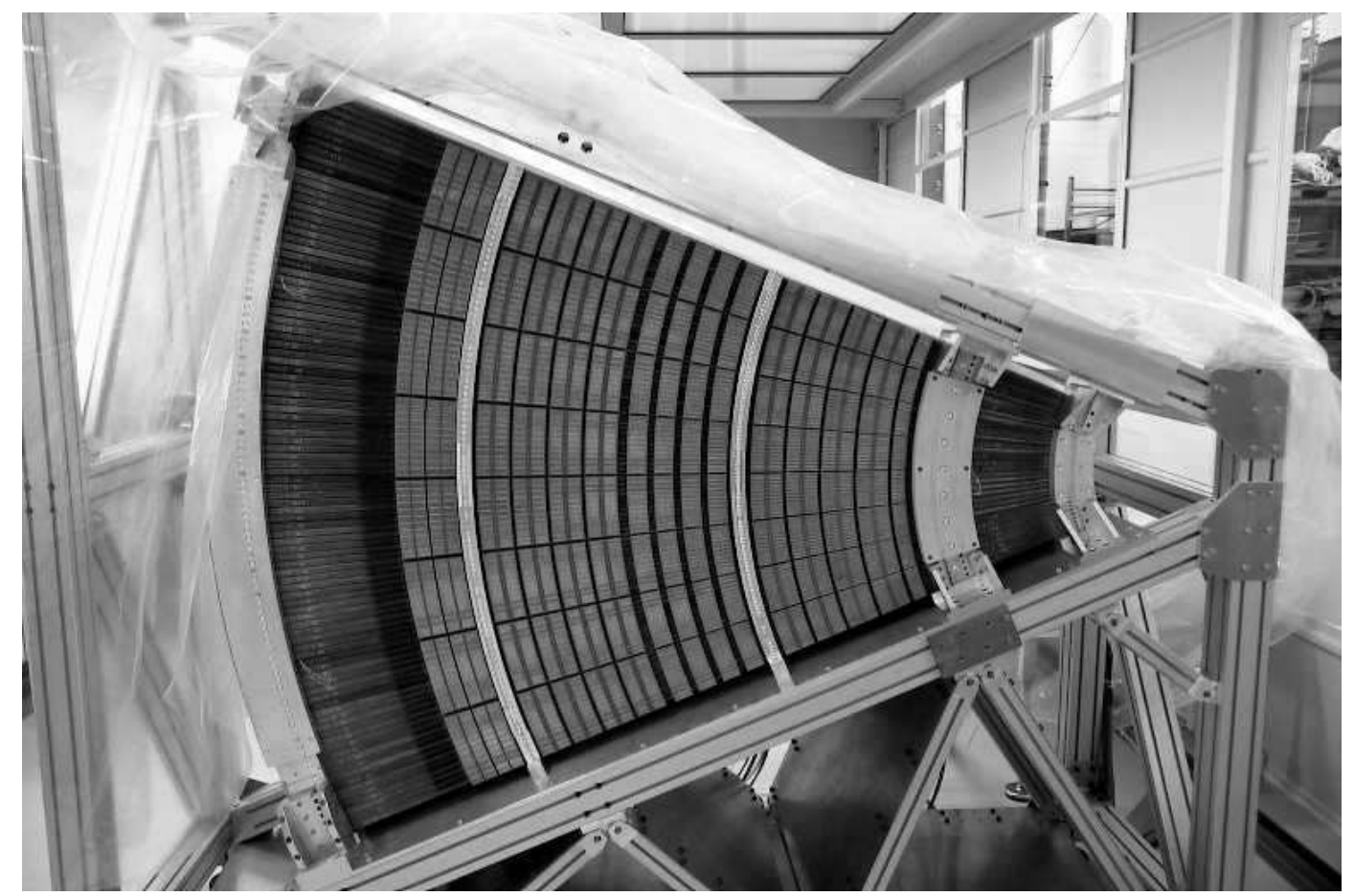

Figure 4. Front view of the first EMEC module, after completion of the stacking, showing some structural elements (the module is held by the stacking frame). All summing boards, except one type (at largest radius), are plugged to the electrodes.

The electrodes are kept centered in the middle of the gaps by spacers, consisting of honeycomb Nomex ${ }^{\circledR 1}$ (paper impregnated with phenolic resin) strips, laid in the flat parts of the accordion (figure 5). The strip thickness varies along the radius following the liquid argon gap.

The calorimeter structure is composed of six support rings: three on the front and three on the back. The total weight of the detector is supported mainly by the two large external rings.

As in the barrel electromagnetic calorimeter, the precision region in the end-cap calorimeters $(1.5<|\eta|<2.5)$ is divided in depth in three longitudinal compartments. The first sampling (front), about $4.4 X_{0}$ thick (about $6 X_{0}$ including the dead material in front of the calorimeter), is segmented with narrow strips along the $\eta$ direction. It is designed to enhance particle identification $\left(\gamma / \pi^{0}\right.$, $e / \pi$ separation) and to provide a precise position measurement in $\eta[11]$. The projective tower transverse size in the second sampling (middle) is the same as defined in the barrel electromagnetic calorimeter: $\Delta \eta \times \Delta \phi=0.025 \times 0.025$. The third compartment (back) has a twice coarser granularity in $\eta$. Both in the inner wheel and in the low pseudorapidity $(|\eta|<1.5)$ extreme of the outer wheel, the calorimeter is segmented in only two longitudinal compartments and has a coarser transverse granularity. Table 1 summarizes the longitudinal and transverse readout granularity in the electromagnetic end-cap calorimeter as function of the $\eta$ range. The $\Delta \eta$ granularity in the front sampling varies with $\eta$ in order to keep the copper strip width larger than a few $\mathrm{mm}$. The $\Delta \phi$ granularity is obtained by gathering the signals from adjacent electrodes. In the outer wheel, 12 adjacent electrodes are summed for a front cell and 3 for a middle or back cell. In the inner

${ }^{1}$ Nomex ${ }^{\circledR}$, a registered trademark of DuPont Company 


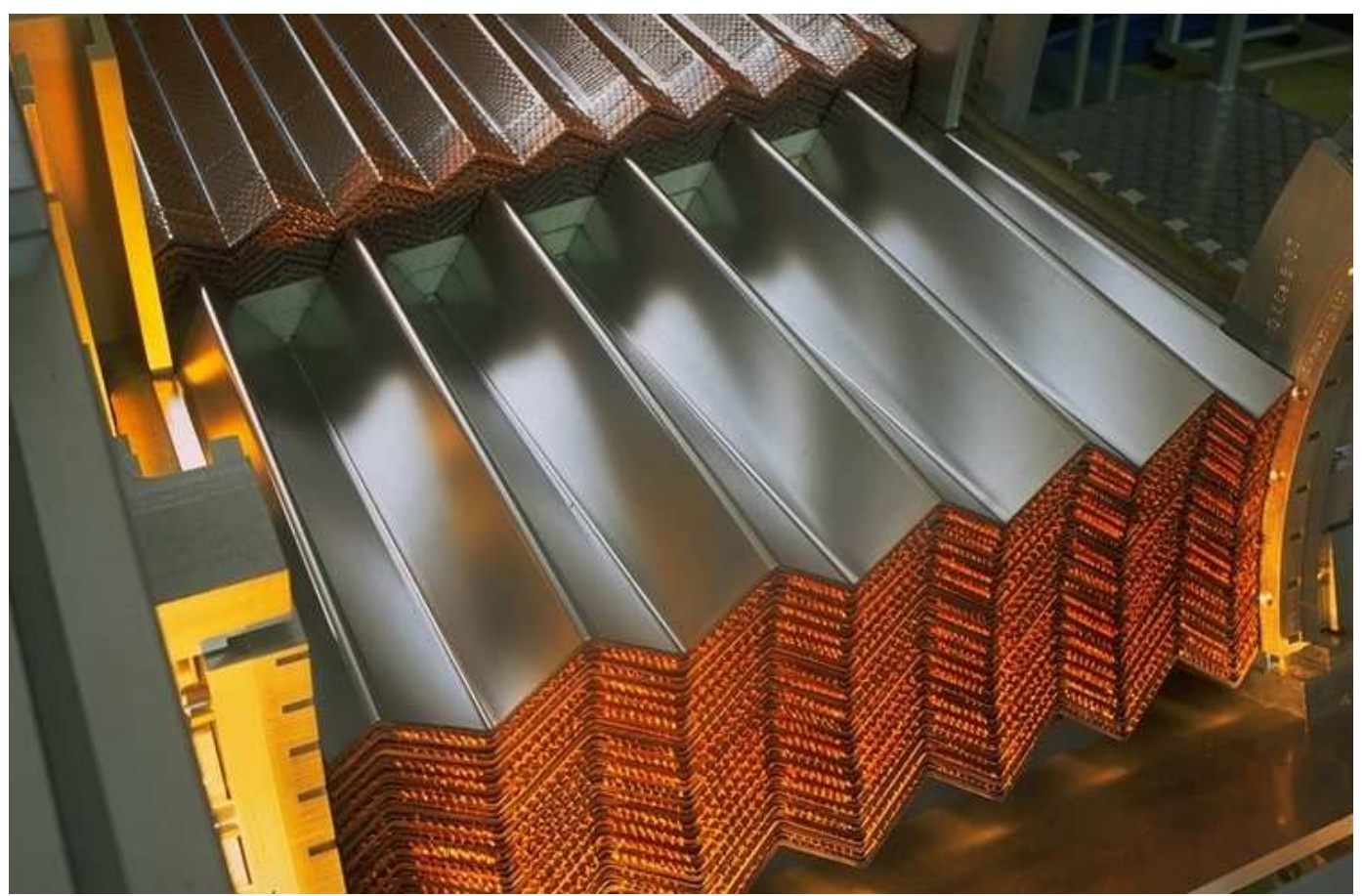

Figure 5. Picture of an electromagnetic end-cap module during stacking, showing the accordion structure of the ATLAS EM calorimeters. The inner wheel is visible in the first plan, with behind a part of the outer wheel. One can notice that the transversal bars of the inner absorbers fill the gap between these two wheels. This light material reduces energy losses in this region. It ensures also an electrical insulation.

Table 1. Longitudinal (front, middle, back) and transverse $(\Delta \eta \times \Delta \phi)$ granularity in the electromagnetic end-cap calorimeter as function of the $\eta$ range.

\begin{tabular}{|cccc|}
\hline$\eta$ range & $\begin{array}{c}\text { Front } \\
(\text { sampling } 1)\end{array}$ & $\begin{array}{c}\text { Middle } \\
\text { (sampling } 2)\end{array}$ & $\begin{array}{c}\text { Back } \\
(\text { sampling 3) }\end{array}$ \\
\hline $1.375-1.425$ & $0.050 \times 2 \pi / 64$ & $0.050 \times 2 \pi / 256$ & \\
$1.425-1.5$ & $0.025 \times 2 \pi / 64$ & $0.025 \times 2 \pi / 256$ & \\
$1.5-1.8$ & $0.025 / 8 \times 2 \pi / 64$ & $0.025 \times 2 \pi / 256$ & $0.050 \times 2 \pi / 256$ \\
$1.8-2.0$ & $0.025 / 6 \times 2 \pi / 64$ & $0.025 \times 2 \pi / 256$ & $0.050 \times 2 \pi / 256$ \\
$2.0-2.4$ & $0.025 / 4 \times 2 \pi / 64$ & $0.025 \times 2 \pi / 256$ & $0.050 \times 2 \pi / 256$ \\
$2.4-2.5$ & $0.025 \times 2 \pi / 64$ & $0.025 \times 2 \pi / 256$ & $0.050 \times 2 \pi / 256$ \\
\hline $2.5-3.2$ & $0.1 \times 2 \pi / 64$ & $0.1 \times 2 \pi / 64$ & \\
\hline
\end{tabular}

wheel, 4 adjacent electrodes are summed for both samplings. Each end-cap calorimeter counts in total 31872 readout channels, including the 768 from the presampler.

Contrary to the barrel part, the drift gap is not constant, but is a function of the radius (R). In the outer wheel it varies from $2.8 \mathrm{~mm}$ at $\mathrm{R}=200 \mathrm{~cm}$ to $0.9 \mathrm{~mm}$ at $\mathrm{R}=60 \mathrm{~cm}$. In the inner wheel it varies from $3.1 \mathrm{~mm}$ at $\mathrm{R}=70 \mathrm{~cm}$ to $1.8 \mathrm{~mm}$ at $\mathrm{R}=30 \mathrm{~cm}$. The detector signal is proportional to the sampling fraction and the drift velocity, and inversely proportional to the liquid argon gap 
Table 2. Nominal high voltage values in liquid $\operatorname{argon}$ as function of $\eta$ in both outer and inner wheels.

\begin{tabular}{|ccc|}
\hline$\eta$ range & High Voltage (in V) & HV board type \\
\hline $1.375-1.5$ & 2500 & $\mathrm{~F}$ \\
$1.5-1.6$ & 2300 & $\mathrm{~B} 1$ \\
$1.6-1.8$ & 2100 & $\mathrm{~B} 2$ \\
$1.8-2.0$ & 1700 & $\mathrm{~B} 3$ \\
$2.0-2.1$ & 1500 & $\mathrm{~B} 4$ \\
$2.1-2.3$ & 1250 & $\mathrm{~B} 5$ \\
$2.3-2.5$ & 1000 & $\mathrm{~B} 6$ \\
\hline $2.5-2.8$ & 2300 & $\mathrm{~B} 7$ \\
$2.8-3.2$ & 1800 & $\mathrm{~B} 8$ \\
\hline
\end{tabular}

thickness. As sampling fraction depends on the gap thickness, their variations along the radius partially compensate each other. But the drift velocity depends on the electric field strength, which for a given high voltage varies with the gap. Thus to get an $\eta$-independent detector response we would need a high voltage continuously varying with $\eta$ (open circles in figure 6). In practice, such a variable high voltage could not be implemented. It is approximated with a variation by steps (closed triangles in figure 6). Table 2 explicitly gives the nominal high voltage values as function of the $\eta$ range, defining the 7 (2) HV sectors of the outer (inner) wheel.

\section{Mechanical components of a module}

\subsection{Presampler}

Each end-cap presampler consists of 32 identical azimuthal sectors (modules). The isometric view of the end-cap presampler sector and its principle design are shown in figure 7 and figure 8 . It is placed in a $5 \mathrm{~mm}$ deep cavity machined in the back side of the cryostat wall.

The granularity of the presampler is $\Delta \eta \times \Delta \phi=0.025 \times 0.1$, i.e. the same as in middle sampling of the calorimeter in the $\eta$ direction and 4 times coarser in $\phi$. This corresponds to 768 readout channels for each end-cap.

One end-cap presampler module consists of $2 \mathrm{~mm}$ thick active liquid argon layers, formed by three electrodes parallel to the front face of the EM end-cap calorimeter. The electrodes, made from double sided printed circuit boards, are separated by honeycomb spacers and glued together at the inner and outer radius with $2 \mathrm{~mm}$ thick G10 bars. The total thickness of the presampler is $9.5 \mathrm{~mm}$.

A negative high voltage is applied to the external electrodes. The signals are read out from the central electrode segmented into pads connected by pins to the $50 \Omega$ strip readout lines printed on the top external electrode. The strip lines are connected at the outer radius of the module to the $50 \Omega$ coaxial cables which lead the signals to the feedthroughs.

The same signal, calibration and HV cables as for the end-cap calorimeter are used. There are two HV cables per one presampler module - left and right part of presampler are fed separately. To increase robustness top and bottom electrodes are connected to HV cables through separate $1 \mathrm{M} \Omega$ 


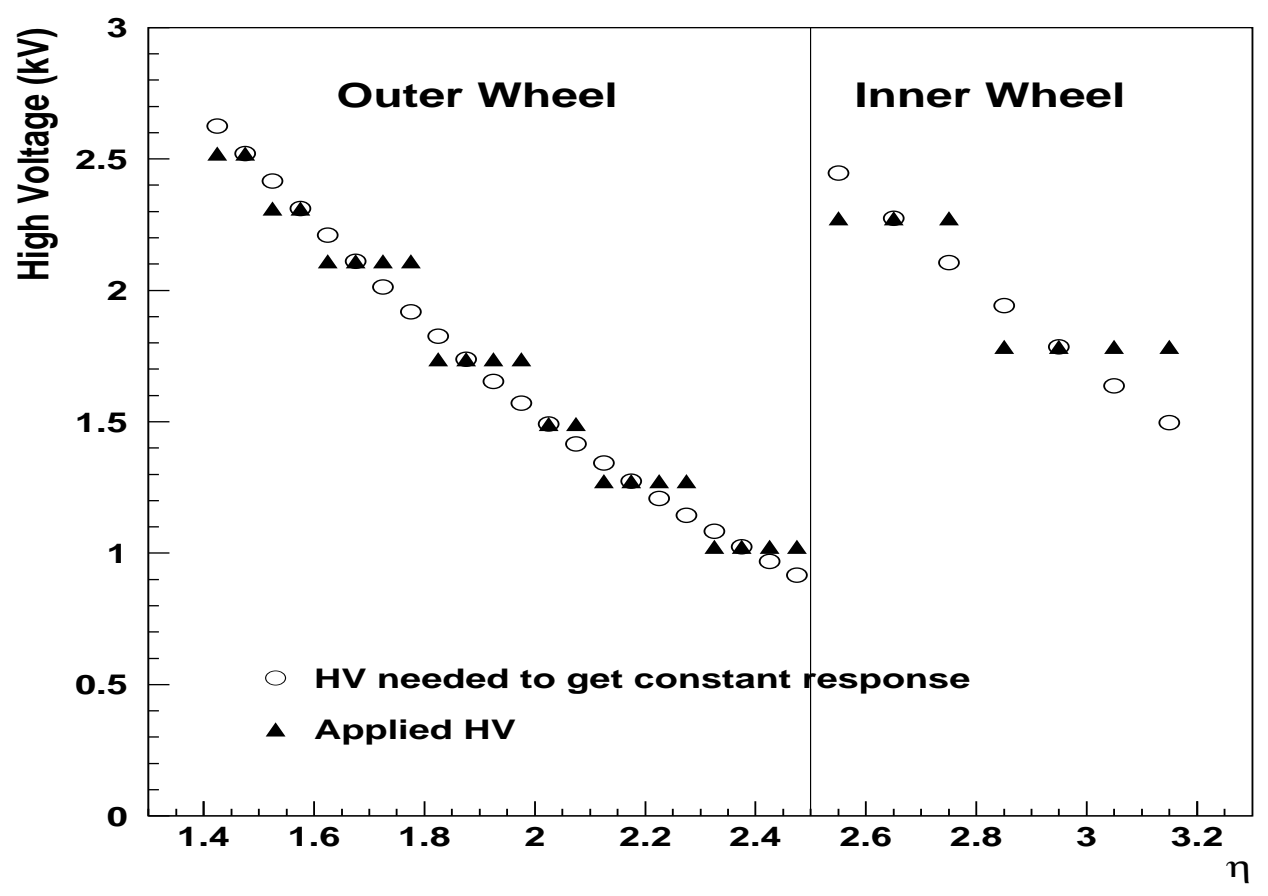

Figure 6. EMEC high voltage distribution. An uniform calorimeter response would need a continuously varying high voltage along $\eta$ (open circles). Actually it is approximated by discrete values (solid triangles).

resistors. Three calibration lines (one per 8 channels) are connected to the signal pads through calibration $3.48 \mathrm{k} \Omega$ resistors ( $1 \%$ tolerance).

The measurements of resistances between HV lines, signal pads and ground planes were carried out before and during module assembling. Final HV tests were performed both in warm state (at $1 \mathrm{kV}$ ) and in liquid nitrogen (at nominal value of $2 \mathrm{kV}$ ). To be qualified the leakage current should not exceed $100 \mathrm{nA}$ and $1 \mathrm{nA}$ respectively.

Details of the presampler construction and performance in test beam are presented in [12].

\subsection{Absorbers}

The requirement to have a constant term in energy resolution better than $1 \%$ imposes very stringent criteria on the design and construction of the calorimeter. Critical items are the uniformity of passive and active calorimeter materials along the particle shower development direction, i.e. the uniformity of the lead thickness and the liquid-argon gap.

Simulations have shown that Gaussian local lead-thickness non-uniformities of the order of $1 \%$ r.m.s. (i.e. $\approx 17 \mu \mathrm{m}$ ) will contribute to the constant term by an amount low enough to keep the constant term under tolerances. A similar result was obtained if the local non-uniformities were distributed uniformly around $\pm 1.8 \%$ of the nominal value (i.e. $\approx 30 \mu \mathrm{m}$ ). On the other hand, and also from physics considerations, local non-uniformities of the liquid-argon gap should be kept below $50 \mu \mathrm{m}$. This restriction constrains both the reproducibility of the geometry of the absorbers and of their relative positions inside the detector. 


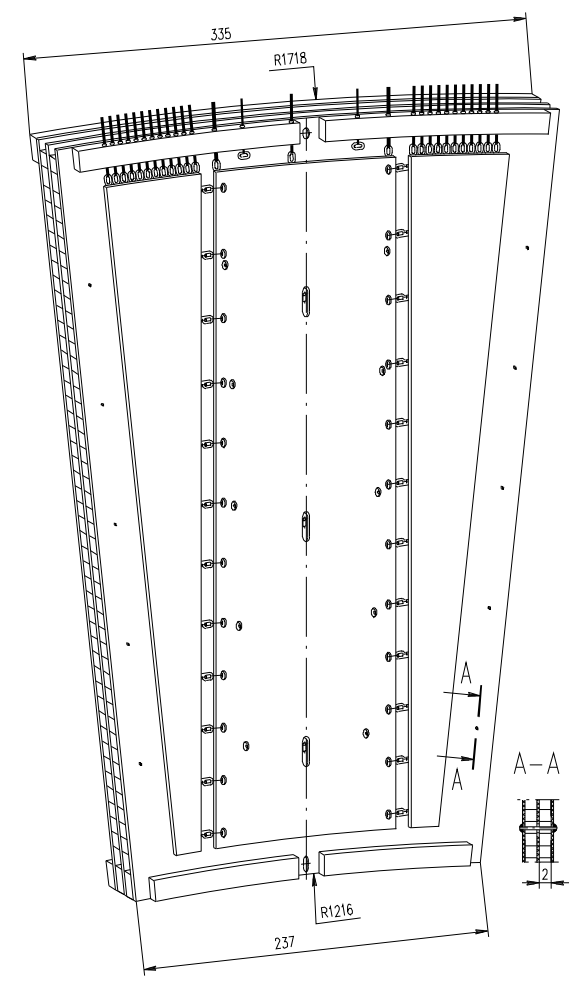

Figure 7. Isometric view of the presampler module.

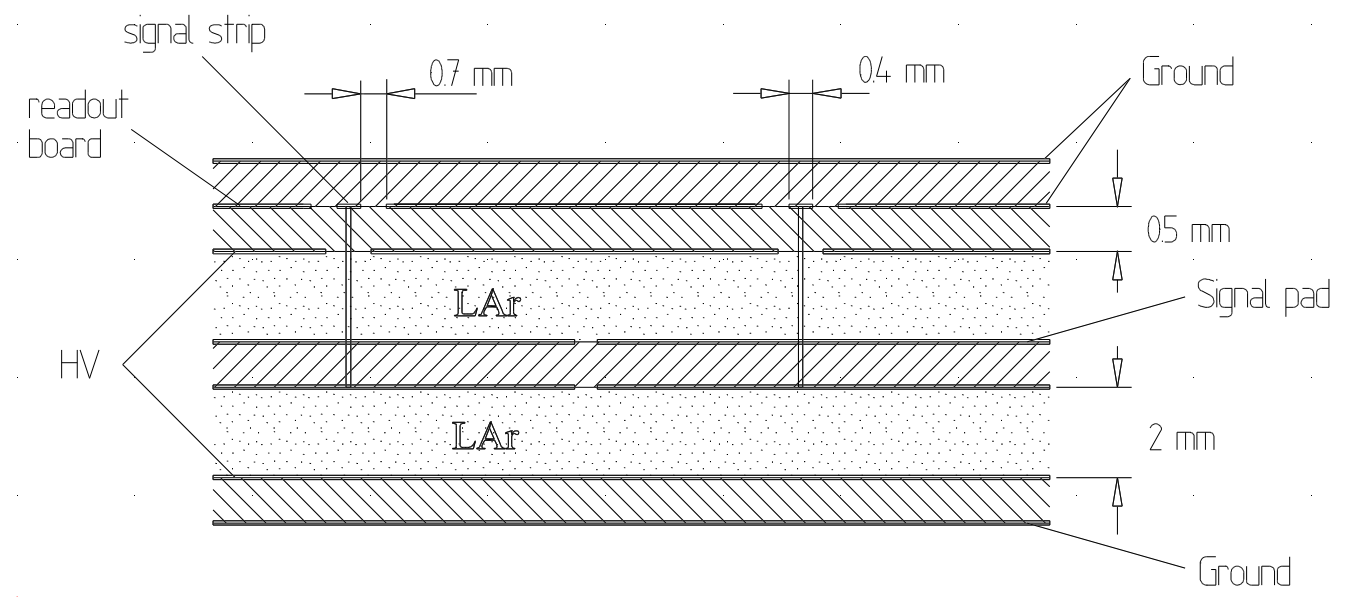

Figure 8. Cross section of a presampler module, showing its principle: $2 \mathrm{~mm}$ thick liquid argon gaps, high voltage distribution, signal collection and grounding.

As the lead is a soft material, it is not possible to maintain an accordion shape without reinforcement. This is provided by two stainless steel skins glued on both faces of lead plates by a layer of glass-fiber prepreg adhesive. In both wheels, the stainless-steel skins are $0.2 \mathrm{~mm}$ thick and the prepreg layers are $0.15 \mathrm{~mm}$ thick.

To link with a good accuracy the absorbers to the structure, we use structural bars on both sides, parallel to the waves. To ensure a good positioning and a stiff junction between absorbers 


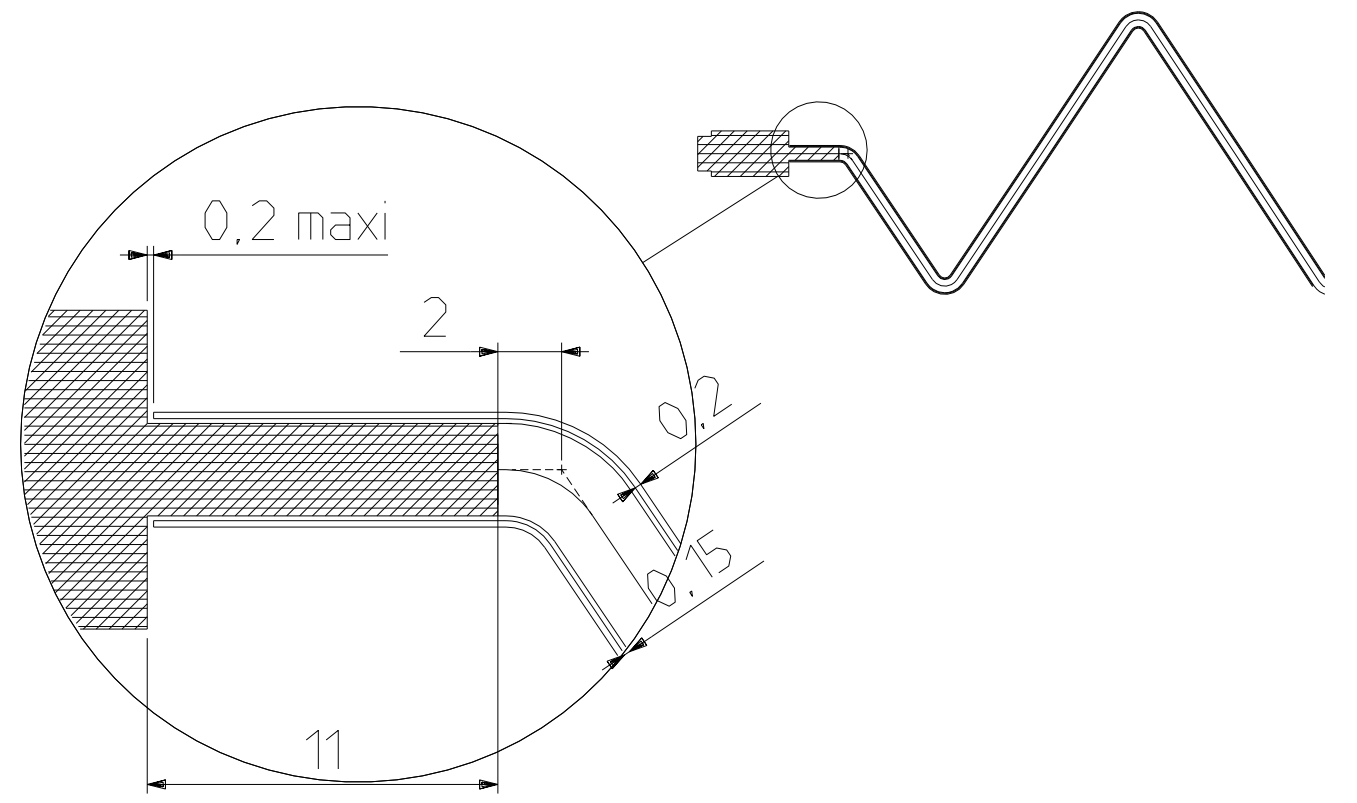

Figure 9. Gluing of a longitudinal bar along the edge of an absorber: the lip of the bar is glued in between the two stainless steel sheets.

Table 3. Geometrical characteristics of the absorbers at liquid-argon temperature.

\begin{tabular}{|lrr|}
\hline Parameters & Inner wheel & Outer wheel \\
\hline Number of waves & 6 & 9 \\
Wave length & $85 \mathrm{~mm}$ & $57 \mathrm{~mm}$ \\
Lead thickness & $2.2 \mathrm{~mm}$ & $1.7 \mathrm{~mm}$ \\
Lead width & $514 \mathrm{~mm}$ & $514 \mathrm{~mm}$ \\
Width of the flat composite sides & $11 \mathrm{~mm}$ & $11 \mathrm{~mm}$ \\
Width of the bar body & $20 \mathrm{~mm}$ & $20 \mathrm{~mm}$ \\
Total width & $576 \mathrm{~mm}$ & $576 \mathrm{~mm}$ \\
Front-edge lead length & $308 \mathrm{~mm}$ & $1387 \mathrm{~mm}$ \\
Back-edge lead length & $351 \mathrm{~mm}$ & $1336 \mathrm{~mm}$ \\
\hline Total weight & $7.5 \mathrm{~kg}$ & $26.0 \mathrm{~kg}$ \\
\hline
\end{tabular}

and bars, the later have a flat part (hereafter called lip), with the same thickness as the lead, which is glued during the absorber curing process between the same stainless steel foils, edge to edge with the lead (figure 9). We also added a third bar, transversal to the absorber, at the edge where the folding angle is most acute. Its purpose is to minimize the deflection due to the sag of the absorber, and to control thermal deformation of wave angles.

The shape of the absorbers is defined at liquid-argon temperature. The procedure followed to compute the shape and the dimensions of each individual component (lead plates, stainless steel sheets, prepreg sheets and bars) at room temperature is based on measurements and computations. Table 3 gives the geometrical characteristics of the absorbers at liquid-argon temperature. 


\subsubsection{Lead production}

The lead for the EM end-cap was produced by rolling, following the same procedure and monitoring than for the barrel calorimeter [8]. The raw material composition is Pb 99.94 DIN 1719. The lead was delivered in rectangular plates $1504 \times 1005 \times 1.7 \mathrm{~mm}^{3}$ for the outer absorbers and $1504 \times 1005 \times 2.2 \mathrm{~mm}^{3}$ for the inner absorbers (three inner absorbers are cut from the same rectangular plate).

To maximize reproducibility, the lead for the whole detector, around 60 tons in total, was produced in one single run. As a consequence, a large fraction of the lead plates have been stored during several years before processing. No hints of aging effects which could have modified significantly mechanical properties (mainly hardness and amorphousness) have been noticed.

The rectangular lead plates were cut to the required absorber dimensions by means of a computer controlled $\mathrm{CO}_{2}$ laser beam. The accuracy of the beam position was $\pm 100 \mu \mathrm{m}$ along distances of a few meters. The cut plates were then cleaned of lead oxide and other dust deposits by an automatic surface brushing machine (the brush was a Scotch-Brite with a $220 \mathrm{~g}$ equivalent roughness). ${ }^{2}$ The absorber curing had to be done within 48 hours after the cleaning of the lead to prevent new oxide formation which could jeopardize its bonding to the stainless steel sheets.

Before cutting, the rectangular plates were optically inspected for scratches and other imperfections; a serial number was given and written permanently on the lead for back-tracking purposes. This serial number was inherited by the absorber built with this lead.

The thicknesses of the lead plates were mapped $\left(50 \times 200 \mathrm{~mm}^{2}\right.$ grid $)$ at $\mathrm{UAM}^{3}$ by a robotic set-up with an ultrasonic probe [13]. These measurements were found in good agreement with the specifications for both large and small lead plates with an accuracy better than $10 \mu \mathrm{m}$.

\subsubsection{Stainless steel sheets}

The plates used for cladding the lead are made of AISI-304-L (surface finished 9F) type stainless steel, with $\mathrm{a} \pm 6 \mu \mathrm{m}$ thickness tolerance guaranteed by the manufacturer. ${ }^{4}$ These sheets are cut out so that they fully cover, after bending, the absorbers including the lips of the longitudinal bars (see section 3.2 .3 and figure 9). The cutting was also done by laser beam and the final dimensions of the cut plates were checked, by means of precision pins, at the time of the absorber sandwich stacking. After cutting, the stainless steel sheets were degreased, pickled and passivated. ${ }^{5}$ The material used for sticking the cladding stainless steel sheets to the lead plates and to the bars is a prepreg Brochier $1454 \mathrm{NC} / 50 \% / 759$ VIT. $200,{ }^{6}$ which consists of a resin-impregnated glass fiber cloth.

\subsubsection{Longitudinal bars}

Figure 10 displays both types of absorbers with their longitudinal bars. The thickness of these longitudinal bars varies linearly with the radius: from $5 \mathrm{~mm}$ to $15 \mathrm{~mm}$ for the outer absorbers, and from $7 \mathrm{~mm}$ to $15 \mathrm{~mm}$ for the small ones. Their length is about $1.4 \mathrm{~m}(0.4 \mathrm{~m})$ for the outer (inner)

\footnotetext{
${ }^{2}$ Cutting and cleaning was carried out by Mendiaraiz S.L., Zumaya, Spain

${ }^{3}$ Universidad Autónoma de Madrid, Madrid, Spain

${ }^{4}$ Teledyne-Rodney Metals, France

${ }^{5}$ The whole cleaning procedure was carried out by CHIMIMECA, Grenoble, France

${ }^{6}$ BROCHIER SA, filiale CIBA-GEIGY, France.
} 
absorbers. On one side, each bar bears an outgrowth, called lip, of the same thickness as lead, used to glue the bar to the absorber (figure 9).

The bars are made of a glass-fiber composite material with resin of type CIBA 5052. The choice was made after considerations of transparency (low $Z$ material), electrical insulation, radiation hardness, absence of liquid argon pollution and mechanical properties. Triaxial and tetra-axial glass-fiber fabrics, cut in strips of different lengths, are combined to achieve a constant thermal expansion coefficient of $15.510^{-6} /{ }^{\circ} \mathrm{C}$. This value reasonably matches those of the structure (aluminium, TEC $\left.=1810^{-6} /{ }^{\circ} \mathrm{C}\right)$ and the absorber $\left(\right.$ TEC $\left.=1410^{-6} /{ }^{\circ} \mathrm{C}\right)$. A sample of this material was irradiated in the $\mathrm{SARA}^{7}$ neutron beam, using a setup [14] allowing to control a possible liquid argon pollution. No evidence of pollution was observed during the exposure (equivalent to $1.610^{14}$ neutrons $/ \mathrm{cm}^{2}$ ).

The absorber positioning concept of the EMEC does not require a precise machining of the longitudinal bars: the bars of adjacent absorbers are not in contact. Thus it was possible to produce the raw bars by using a rather cheap process, so-called Resin Transfer Molding (RTM), ${ }^{8}$ with custom molds.

The binding to the structure is achieved by T-shaped heads precisely machined $( \pm 40 \mu \mathrm{m})$ at each end. For the positioning of the absorber to the structure the bars have accurate holes used as geometrical references. They are drilled with respect to the median axis of the lip and to the Tshaped heads with a $\pm 10 \mu \mathrm{m}$ tolerance. ${ }^{9}$ Further details of the attachment of the absorbers to the structure can be found in section 3.3. Each lateral bar also houses the electrode connectors in two (only one in the inner wheel) long notches, $1.2 \mathrm{~mm}$ thick and $7 \mathrm{~mm}$ deep, running along the bar, top and bottom, on the external side of the absorber. Additional machined notches accommodate the solders of the ground contacts on the electrodes (see flap description in section 3.4).

The production rate was half a module (including $10 \%$ of spare), i.e. 53 pairs of large bars and 18 pairs of small ones, per month. The full production took 32 months.

\subsubsection{Absorber fabrication}

The fabrication of one absorber consists of three steps: the stacking of the different plates (lead, stainless steel and prepreg) and of the lateral bars to form a flat absorber sandwich; the bending of this sandwich to the required absorber geometry; and finally, the curing of the prepreg to firmly stick the lead to the stainless steel and bars as well as to provide the final shape, rigid enough, to the absorber.

The main purpose of bending the sandwich is to deform the sandwich locally, into the plastic regime, so as to form the top and valley accordion curves of the absorber with good precision ( $\pm 150 \mu \mathrm{m}$ ), without modifying the properties (in particular thickness) of the rest of the sandwich area.

Given the two different types of absorbers, two different presses are needed. During the bending, the knives follow a circular movement around an axis perpendicular to the absorber symmetry plane (parallel to the jaw plane of the press). This is to deform the sandwich only at the accor-

\footnotetext{
${ }^{7}$ LPSC, Grenoble, France.

${ }^{8}$ Done at Compreforme, Châtillon-sur-Seine, France.

${ }^{9}$ The precision machining (T-shaped heads and centring holes) was done in Talleres Aratz, Vitoria, Spain.
} 


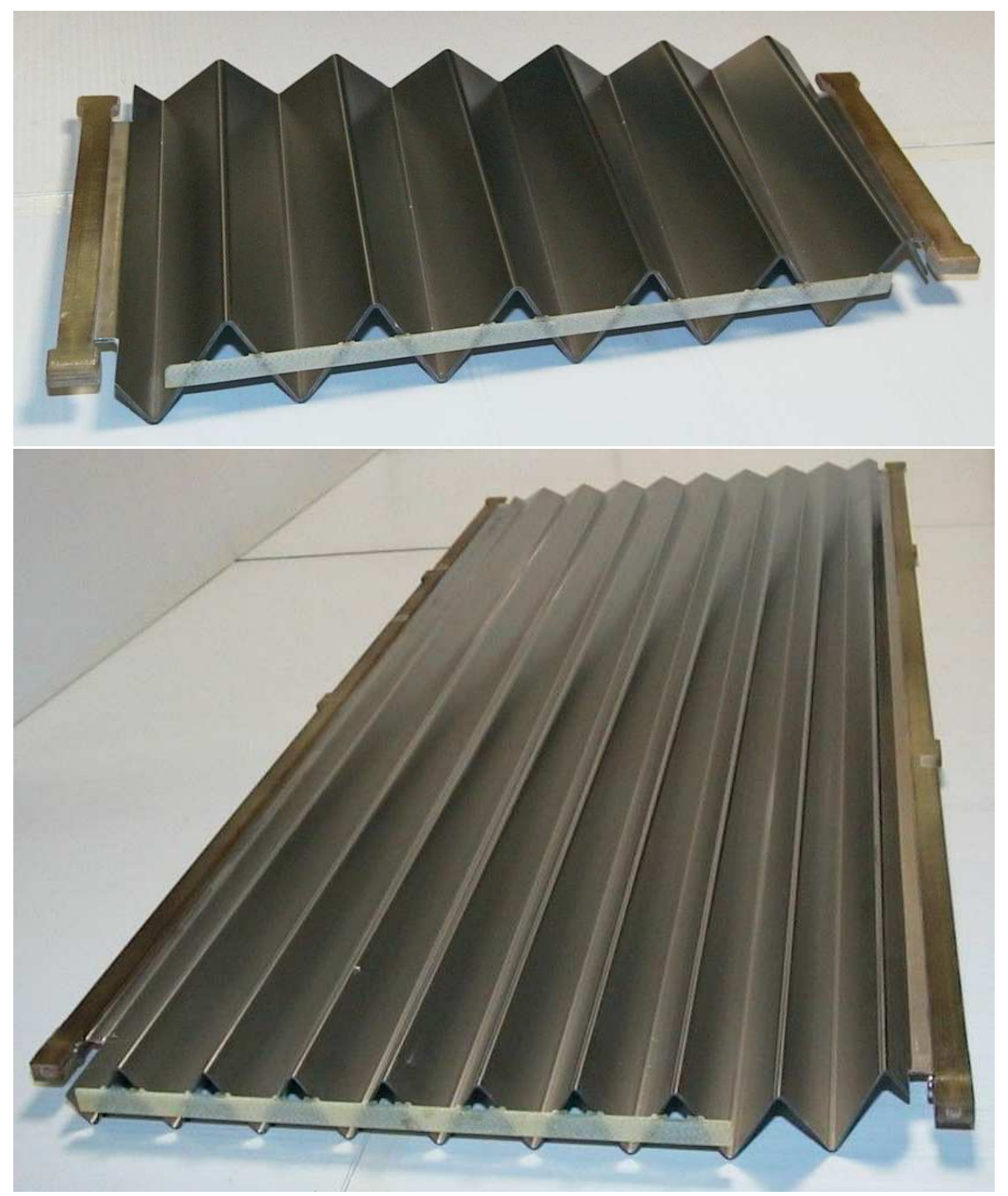

Figure 10. Small (top) and large (bottom) absorbers. On each absorber, the longitudinal bars parallel to the waves and the transversal bar glued on the waves are clearly visible.

dion folds. These circular paths and also the peculiar shape of the knives are consequences of the variation of the folding angle along the absorber.

In addition to the main hydraulic cylinder, the presses feature two lateral hydraulic cylinders (and their corresponding force-transmitting structures) to provide a lateral force at each end of the absorber, once it has been already bent, to compensate force transmission losses from the main hydraulic cylinder due to the complicated geometry of the absorber. The optimum vertical pressure was estimated to be around $400 \mathrm{kPa}$. These figures translate into vertical (lateral) hydraulic cylinders able to provide total forces of 9 and 30 tons ( 1 and 3 tons) for the inner- and outer-absorber presses, respectively.

For the polymerization of the prepeg inside the absorber, the bent sandwich is placed in between two sub-molds (a set of two sub-molds will thereafter be called mold). The shape of these 
molds was computed carefully, in order to get the right geometry of the absorbers in liquid argon, by taking into account all thermal expansions in all the phases of the polymerization cycle.

The prepreg polymerization itself occurs during a custom temperature-pressure cycle provided by an Air-Industry autoclave ${ }^{10}$ to the bent absorber inside the mold. The autoclave was large enough to perform the molding/curing in batches of 10 outer absorbers and 4 inner absorbers. This feature speeded up considerably the production process but introduced the necessity of an extremely careful manufacture of the $10+4$ absorber molds in order to guarantee the needed reproducibility on the absorber geometry. This reproducibility was extensively checked by detailed 3D mapping (with a tridimensional measuring machine) of both the molds themselves and the first absorbers coming from the different molds. When necessary, corrections were applied to the molds.

From the 3D measurements of the first absorbers produced with all the molds, a real absorber geometry was defined and used as a pattern for the rest of the production. The quality control of the produced absorbers consisted of an optical inspection for possible damage during production and a comparison of its geometry with that of the pattern.

For $90 \%$ of the absorbers this comparison was coarse, namely the thickness of the absorber was measured at eight points of its contour with a palmer and its width (distance between the lateral bars) was measured at two predefined locations with a custom gauge. If the measurements did not differ from the pattern by more than an established amount ( $40 \mu \mathrm{m}$ for the thickness and $100 \mu \mathrm{m}$ for the absorber width and wavelengths), the absorber was accepted, otherwise it was carefully analyzed before accepting or rejecting it.

For the remaining $10 \%$ of the absorbers, a complete 3D mapping was carried out and thicknesses widths and wavelengths extensively compared with those from the pattern. For each end-cap module produced at least one absorber cured in each mold was measured in such an extensive way. The idea behind this procedure was to monitor the stability of the geometry of the molds during production. No significant deviations have been observed.

\subsection{Mechanical structure}

\subsubsection{General description}

The mechanical structure of each EMEC wheel is composed of six support rings, three on the front and three on the back sides. The total weight of the detector is held mainly by the two large outer rings. They transfer the weight to rails mounted on the cold wall of the cryostat (see section 7 ). They are also equipped with a vertical guiding part, the top key, to ensure the centering of the detector with respect to the cryostat. In order to minimize the width of the crack between the inner and outer wheels, two intermediate rings (back and front) are designed to bind these wheels together. The inner rings are designed to ensure the conical shape of the inner wheel.

The position of each absorber is precisely defined by pins inserted into centring holes drilled in both bars and support rings. The holes drilled in the bars are cylindrical, while they are oblong in the rings. This guarantees a $20 \mu \mathrm{m}$ (including both machining and clearance) positioning accuracy in $\phi$ of the absorbers, and frees the pins of radial stresses.

Two lighter rings, the indexing rings, are added on each side of the outer wheel; they help in minimizing the absorber bar deformation and to control the gap thickness. Of course each of these

\footnotetext{
${ }^{10}$ Fibertecnic, Vitoria, Spain.
} 


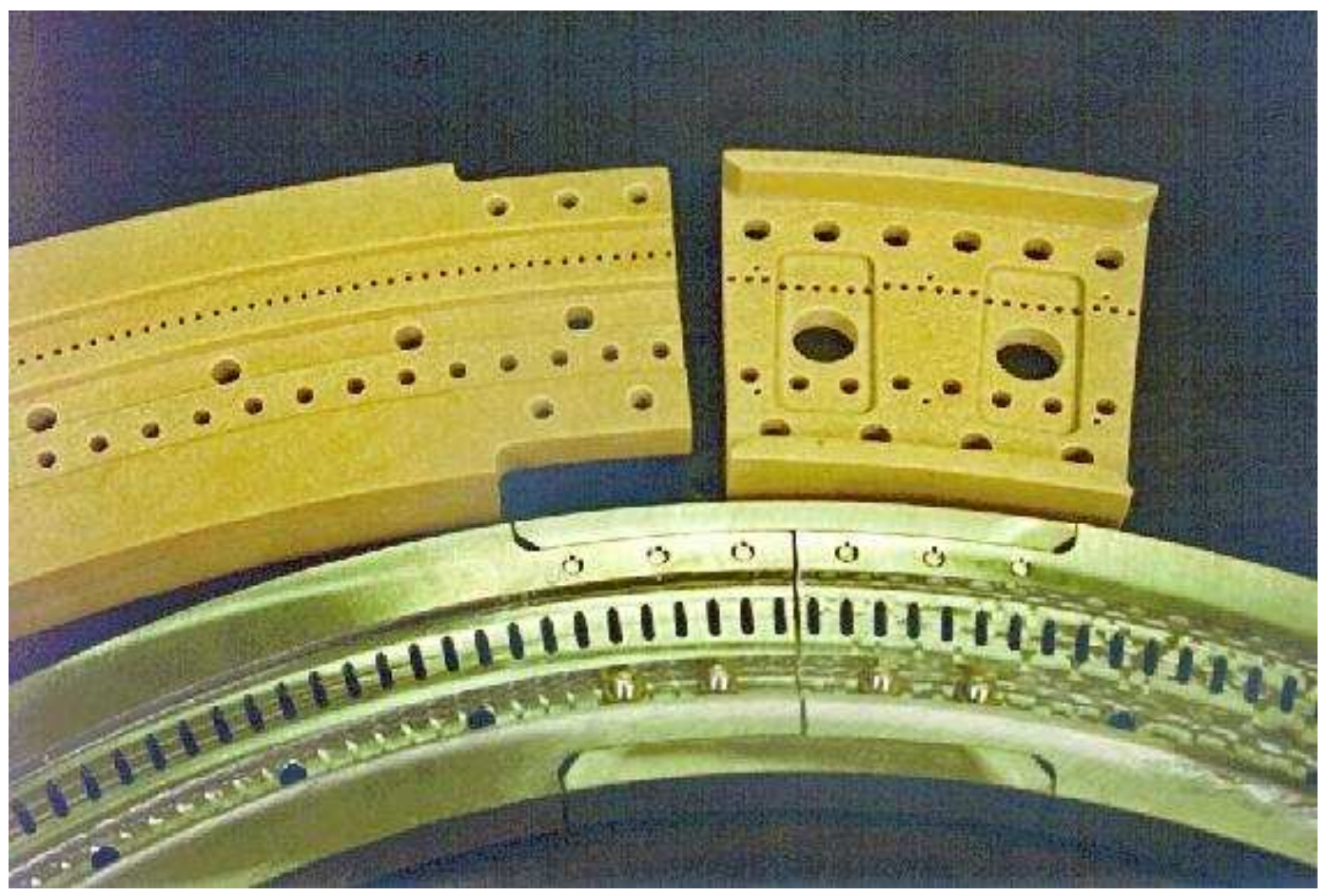

Figure 11. Intermediate and inner rings around the junction areas.

ten rings is divided into eight parts corresponding to the eight modules of an EMEC wheel. The five front rings (three support and two indexing) are visible on figure $\$$.

The modules are connected together by junction elements fitted on the six support rings. As an example, figure 11 shows a picture of intermediate and inner rings around junction areas. In these regions the mechanical inertia of each ring is shared in two almost equal parts: about half of the material is removed from the ring itself and replaced by a U-shaped part. This piece replaces exactly the removed material; by this way the overall cross-section stays identical all along the ring. The length and the inertia of these U-shaped junction elements are computed to keep the detector deformations at an acceptable level. The U-shaped junction pieces are fixed to the rings with screws. Two notches are machined on the flat part of each junction piece. Nuts, corresponding to these notches, are machined on the ring elements. This system ensures the longitudinal transfer of the strengths all along the ring. The junction pieces allow fine tuning of the alignment of the ring parts during the wheel assembly (see section 8.1).

To control the width of the detector, its transverse shrinkage and the parallelism of the front and back faces, 16 stretchers are put between the front and back outer rings, eight being located at the module junctions. The transverse bars glued on the inner absorbers play a similar role between the front and back intermediate rings. The inner rings are linked together by a cone-shaped shell.

Temporary parts replace the junction elements and the stretchers until the module is assembled in the wheel.

To ensure a shrinkage of the calorimeter similar to the cryostat one, most of its structure components (rings and screws) are made out of aluminium alloy, whose mechanical characteristics are carefully specified, e.g. the elastic strength limit Rp0.2 $>450 \mathrm{MPa}$. In order to perform the 
quality control tests, probes having followed the same manufacture cycle as the structure parts have been produced. For this purpose the largest parts have been machined with a small material extension. These extensions have been used for tension and compression tests. In addition, some tension tests performed with good (HRB > 90) and bad material, allowed to calibrate the hardness test. This one, easier, was applied on all the aluminium parts.

In order to minimize the dead material in front of the EMEC, the intermediate front ring is made of a compressed composite, with short glass fibers and epoxy resin. In the plan of the ring, the Young's modulus is $18 \mathrm{GPa}$ and the tensile strength is $280 \mathrm{MPa}$.

The weight of the wheel is supported on two feet which are strongly bolted (14 aluminium $\varnothing 16$ bolts) on the junction stretchers of the horizontal diameter. For the fixation of those stretchers on the rings, the most loaded screws are made of high strength titanium alloy (TA6V). The feet laid on the rails of the cryostat. The supporting surfaces are located within a tolerance of $\pm 0.1 \mathrm{~mm}$ with respect to the median plane of the stretchers.

Mechanical and thermal computations of the structure have been carried out with a complete finite element model of the wheel and its components. The largest vertical deviation with respect to the ideal cylindrical shape was found smaller than $1.4 \mathrm{~mm}$ for the outer radius and $1.8 \mathrm{~mm}$ for the inner radius. The lateral positioning with respect to the cold wall of the cryostat is achieved with a large key inserted in a groove located in the top part of the cryostat. This top key is fixed on the stretcher at the top of the wheel. It allows to keep the wheel well centered in the cryostat during the cooling down.

Reference holes $(\varnothing 10 \mathrm{H7})$, precisely drilled in the six supporting rings, allow to check the geometry of a module. This geometry is controlled by means of calibrated bars. The relative position of two ring elements (e.g. concentricity) is defined by three characteristic distances (e.g. two "radii" and one "diagonal"). Each reference distance is materialized, with a $\pm 0.02 \mathrm{~mm}$ accuracy, by two holes drilled in a calibrated bar. Thus, in total, 12 bars are necessary to define the shape of a module. The same set of calibrated bars was used to check the shape of all modules.

\subsubsection{Outer rings}

The outer rings form the carrying framework of the wheel. The binding of the absorbers to the outer rings differs from the way used with the intermediate and inner rings. An aluminium insert is put in a hole drilled in the head of the bar. It receives two aluminium screws. The first one, parallel to the bar axis, gives the precise $\phi$-position of the absorber. This screw also allows the bar to be stretched. Tuning the stresses of these longitudinal bars allows to conform the geometry of a module (see section $\emptyset$ and section 8.1). The other screw, perpendicular to the bar, is used to press the external edge of the bar head against the internal face of the ring. These contact surfaces are precisely machined ( 0.1 planarity).

At the outer radius, a large absorber is rather flexible. In order to minimize its sagging, an outer central ring, is bound to the stretchers. By this way the middle of the outer edge of an absorber is positioned by a stainless steel pin going through this outer central ring and inserted in a hole drilled in the transversal bar of the absorber.

\subsubsection{Intermediate and inner rings}

The intermediate rings rigidly link the two wheels and guarantee the crack width of $3 \mathrm{~mm}$. They 
consist of two parts which form T-shaped grooves intended to receive the ends of the bar. During the stacking, in order to slide the bars inside the grooves, the screws linking both parts are not tightened. The absorbers are positioned in $\phi$ by the pins ( $\varnothing 2$ and $\varnothing 5)$ made of stainless steel on the back face, and of carbon fiber/epoxy on the front face. When the stacking of a module is completed the heads of the bars are squeezed by pressing together both parts of the grooves by screws.

As the glass fiber composite is not isotropic, various tests were performed in order to characterize the mechanical and thermal properties of the material. The results have been used to define the acceptable loads during the cooling down and the warm up of an end-cap (see section 9.2).

The absorbers are positioned in a similar way within the inner rings with a T-shaped extremity in a groove and indexed in $\phi$ by stainless steel pins. The radial clearance provided by the oblong holes is larger $(2.8 \mathrm{~mm})$ to let the ring free to move under thermo-mechanical loads. In addition the heads of the bars are not squeezzed, allowing a clearance of $0.2 \mathrm{~mm}$. A cone-shaped stretcher gives the width of the wheel. It consists of a glued structure comprising a skeleton and two rolled skins. The gluing frame guarantees the width within a tolerance of $\pm 0.2 \mathrm{~mm}$.

\subsubsection{Indexing rings}

In order to minimize sagging effects and to keep the gap between absorbers as uniform as possible, the outer absorber bars are also fixed to two indexing rings located at $1 / 3$ and $2 / 3$ of the bar length. Those rings are drilled with precise oblong holes. In order to minimize the dead material in front of the calorimeter the cross-section of these rings is rather thin and the absorbers are positioned with aluminium pins.

\subsubsection{Production}

At the prototyping phase, two sectors and one junction piece of each ring have been fabricated for qualification. The large parts and the intermediate front rings have been produced by industry. The smallest parts have been machined at CPPM. ${ }^{11}$

All the aluminium parts of the detector have then been produced by the BINP at Novosibirsk. All the other parts (mainly the composite ones) have been produced by industry. ${ }^{12}$

\subsection{Electrodes}

The readout electrodes are large polyimide-copper printed circuits, bent to fit the absorber geometry. They consist of a double sided copper clad polyimide glued on a single sided copper clad polyimide. The overall thickness after gluing is $275 \mu \mathrm{m}$, but the structure is not perfectly symmetric. The external copper layers are used to apply high voltage through liquid argon gaps, while the induced electrical current is read on the inner one.

The desired granularity in pseudorapidity and in depth is achieved by etching the 3 copper layers. Connection lines and HV buses are obtained in the same way. These are drawn on front and back electrode edges, outside the active region.

As for the absorbers, the two wheels are equipped with different types of electrodes. Flat electrode drawings are shown in figure 12.

\footnotetext{
${ }^{11}$ Centre de Physique des Particules de Marseille, Marseille, France.

${ }^{12}$ Talleres Aratz, Vitoria, Spain.
} 


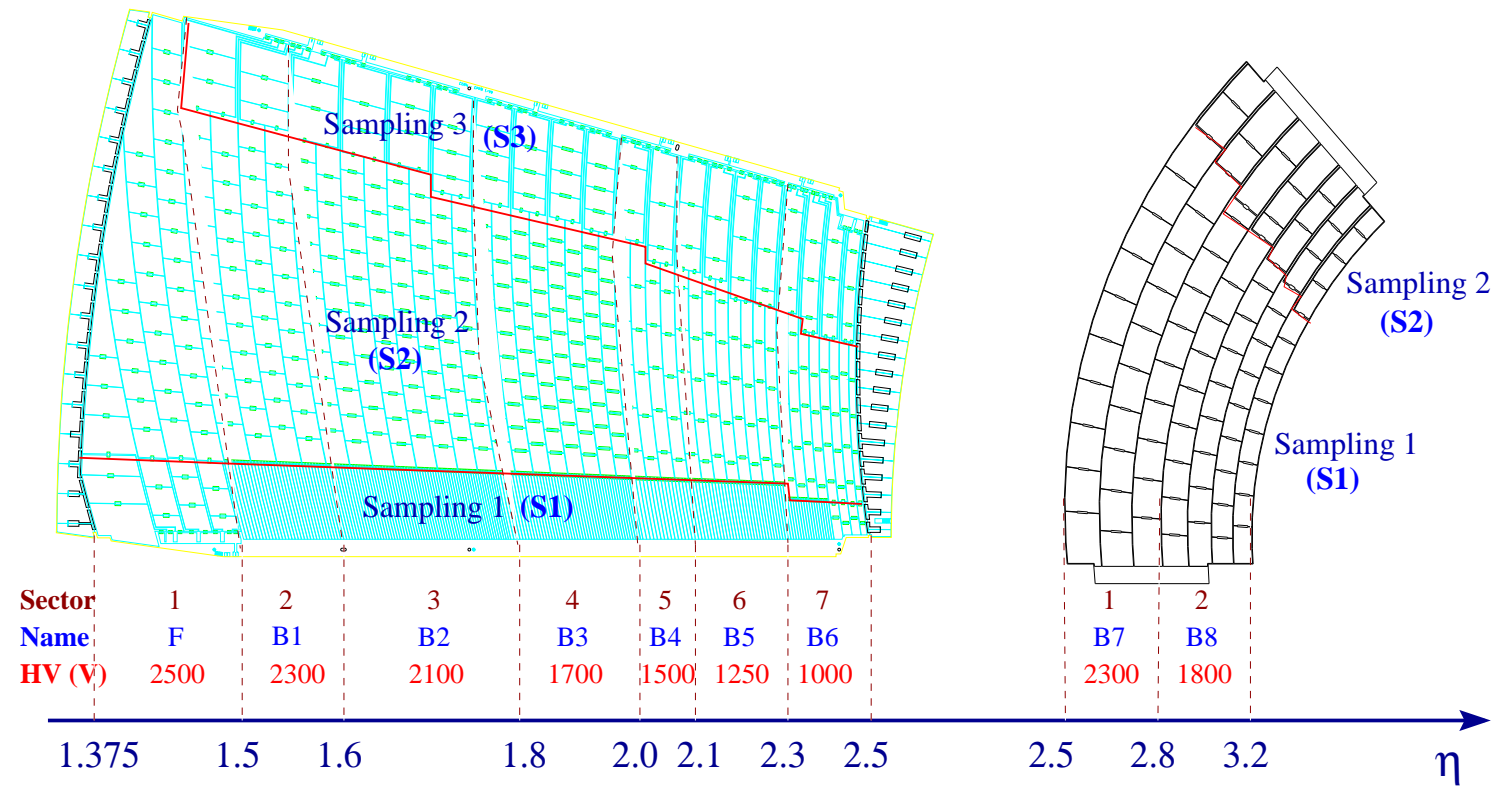

Figure 12. Outer and inner wheel flat electrode schematic views. Sampling regions are represented as well as high voltage sectors. High voltage values are indicated for each sector at liquid argon temperature.

The outer electrodes have three longitudinal samplings. The first one extends till 6 radiation lengths $\left(X_{0}\right)$ including material in front of the active volume. The second sampling ends at $24 X_{0}$ and the third sampling covers the remaining volume, except for $|\eta|<1.5$, where the second sampling extends up to the end of active volume. The inner electrodes have only two samplings, separated at $24 X_{0}$.

The fine $\eta$ granularity of the first sampling in the outer wheel has been optimized in order to discriminate $\pi^{0}$ from photons. The $0.025 / 8$ pitch could not be achieved for $|\eta|>1.8$, because this would result in strip width below $5 \mathrm{~mm}$. Beside becoming too narrow as compared to shower width, even close to the conversion point, this would be a source of high cross-talk. Thus the granularity of first sampling varies (table 1). The granularity in $\phi$ is achieved by gathering the ouputs of neighbouring electrodes.

The electrical ground is brought from the cryostat wall at the signal feedthroughs via the signal cables to electrode ground pads. This ground has to be routed to absorbers, which serve as cathodes in the liquid argon gaps. The absorbers are isolated from the cryostat, which is itself isolated from its supporting structure (Tilecal extended barrel). The ground contact is carried by a single sided gold plated copper polyimide sheet (the flap), put across the gap. The gold plated copper touches the absorber on one contact line while it is soldered to the electrode ground pads on the other side. The flap is held in contact against the absorber by the spring effect of a polyimide tube which is lightly compressed across its diameter (figure 13). Seven different tube diameters are necessary to achieve contacts all along the $\eta$ direction.

High voltage is distributed to the cells of the two external layers through silkscreened epoxy ink resistors of about $1 \mathrm{M} \Omega$, with some redundancy where possible. The HV layers of sampling 2 and 3 are divided into longitudinal pads, connected by silk screened resistors. Their numbers 


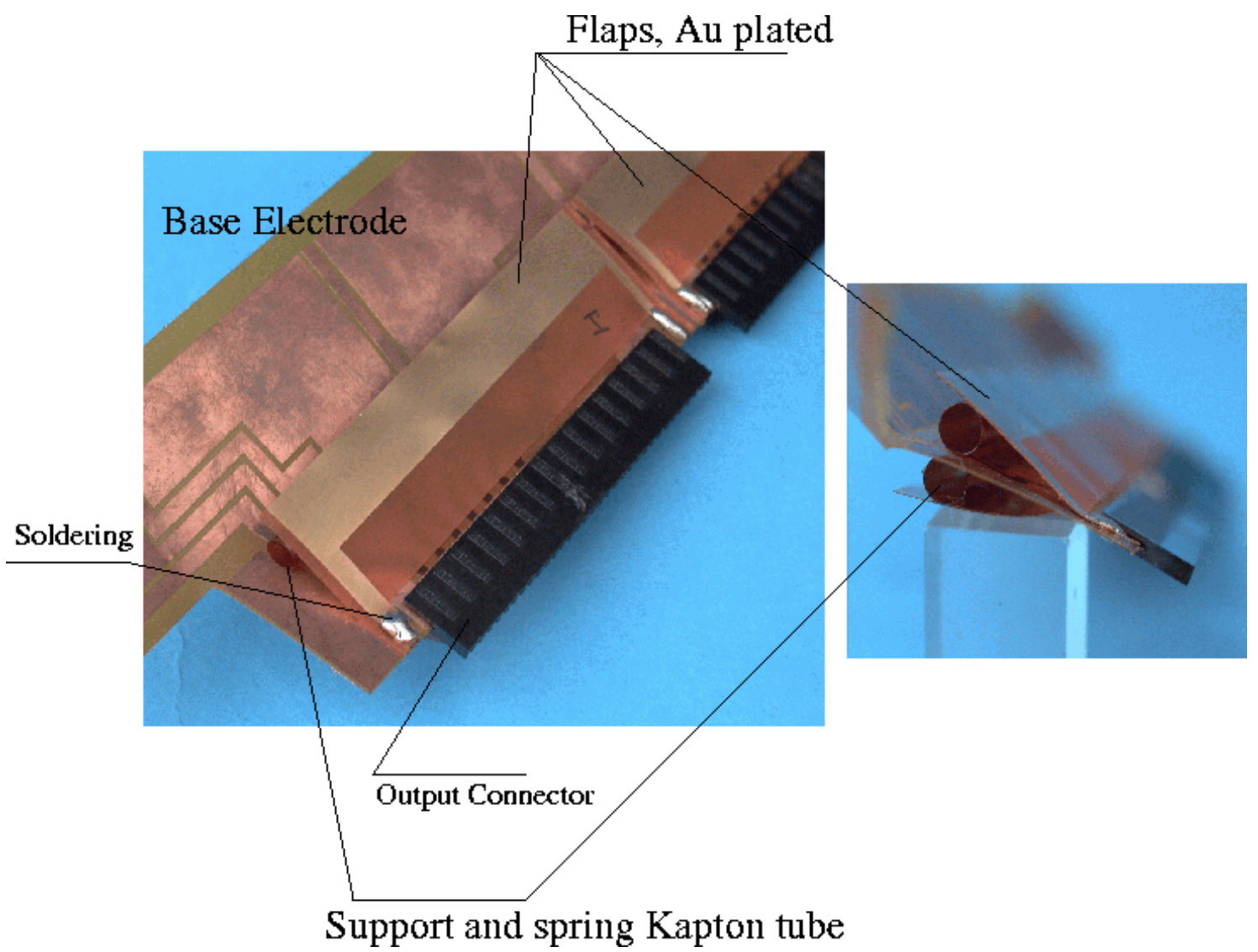

Figure 13. Ground contact scheme.

and values are a compromise between the preamplifier protection from accidental sparking (high resistance values) and the minimization of the impact of the space charge from pile-up events (low resistance values). A typical value is few hundreds of $\mathrm{k} \Omega[15]$.

In order to get the desired angle variation along the accordion folds, electrodes have been bent by using a single tilted knife (one for each electrode type) pushing them against an elastic rubber foam plate. The tilt angle had to be tuned for each electrode batch (using test electrodes).

Electrodes have been tested all along the different steps of their fabrication and equipment. Visual inspections, resistor and capacitance measurements, high voltage tests have been performed on all electrodes, using dedicated test benches. Some resistors showed a larger value after bending, and have been repaired. More details on the electrodes fabrication and tests can be found in [10].

\subsection{Spacers}

The spacers keep the electrode centered in the argon gap between the absorbers. As shown in figure 14, the accordion shape of spacer nets is obtained by sticking together Nomex ${ }^{\circledR}$ strips by the means of impregnated glass-fiber wires. ${ }^{13}$ Three (two) different types of nets are needed to cover an outer (inner) absorber. To avoid damaging the electrodes, the wire is always on the absorber

\footnotetext{
${ }^{13}$ Vicotex SXME M27/28\%/EC9 4x68
} 


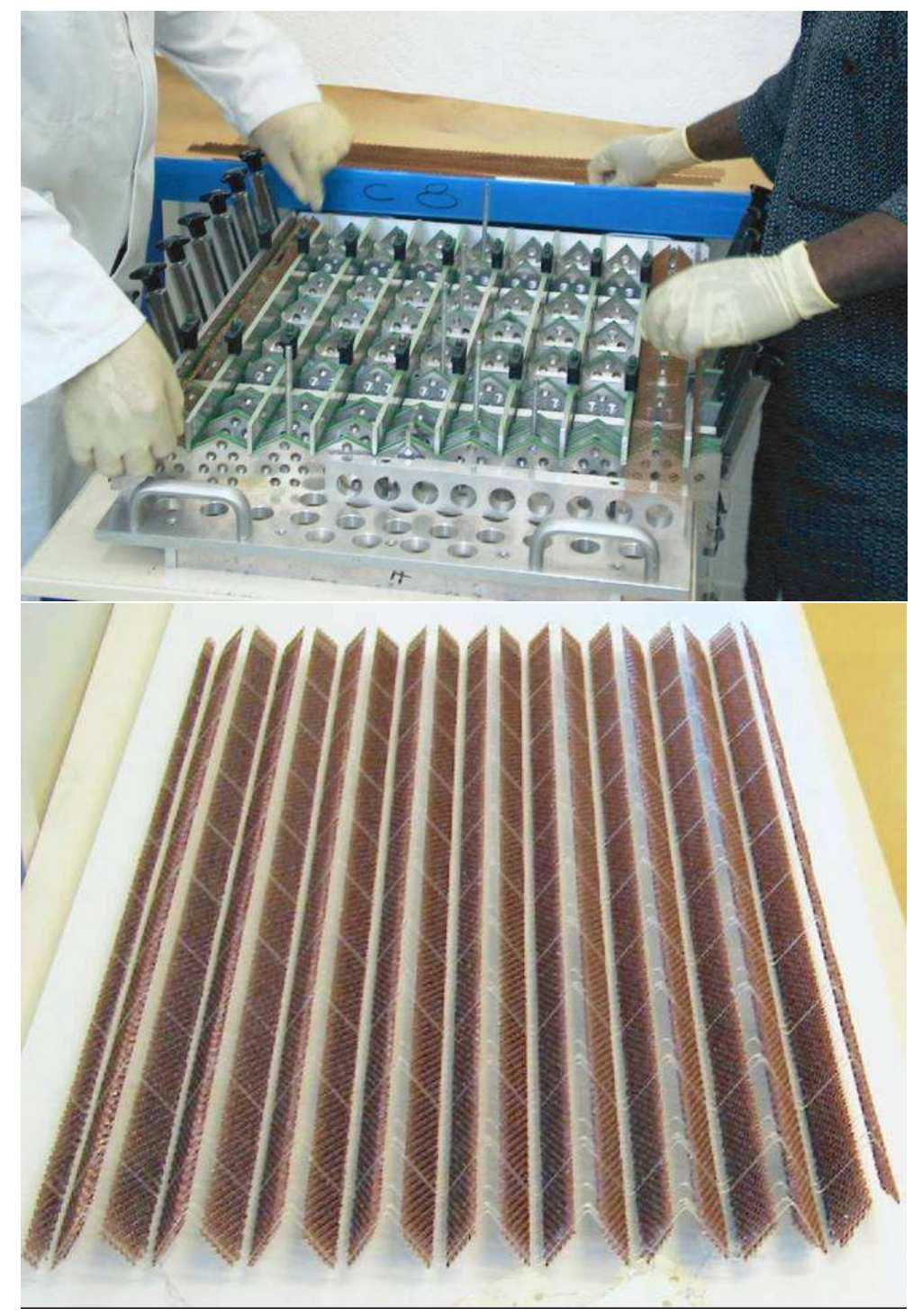

Figure 14. Top: one of the 10 molds for spacer fabrication. Bottom: one of the 10 types of EMEC spacer nets.

side. Thus a type of nets for each side is necessary and in total 10 types of spacer nets had to be produced.

Nomex ${ }^{\circledR}$ paper impregnated with phenol-formaldehyde resin and with a honeycomb structure was produced and cut into sheets by Hexcel Composites in Belgium. To match the gap thickness variation along $\eta$, those sheets have a linearly variable thickness, with a very tight tolerance of $+0.05 \mathrm{~mm}$ and $-0.25 \mathrm{~mm}$. During the manufacture, the thickness was cross-checked on each sheet by the company then again at CPPM. For the thinnest sheets, up to $30 \%$ of delivered batches were rejected for noncompliance with these specifications.

The spacer nets were produced at CPPM. The sheets were cut into trapezoidal strips to be put in the flat part of the accordion straight sections. A specific tooling has been developed to get appropriate cutting. Ten molds, as shown in figure 14, were used to get the appropriate profile for 
each net type. The transversal wires were pressed on the honeycomb strips by mean of knives of different angular shapes to match the variable angle geometry of the absorbers. Wires were cured at $130^{\circ}$ by heating molds in an oven for an hour. Three to four cycles were done a day, each cycle producing ten nets. Spacers were all controlled visually, in dimension and on 12 spots in thickness. Then they were cut to their final shapes, cleaned of dust by blowing dry air and packed.

Just before stacking, each spacer was tested between two absorbers at a voltage $50 \%$ higher than nominal. Either the spacer was accepted for stacking or was cleaned again and tested back. From the Nomex ${ }^{\circledR}$ sheet production up to the stacking of the spacers, strong care was taken to control the humidity level as well as the cleanliness. By the end of the production, only a few percent of produced spacers were routinely rejected. Thanks to such drastic procedures, only very few channels did not hold nominal high voltage during stacking.

\section{Module assembly}

The EMEC modules were stacked in two sites: CPPM and UAM, from August 2001 to March 2004. In both sites the stacking took place in a temperature, humidity $(<50 \%)$ and pressure controlled clean room.

The stacking frame (figure $\bigoplus$ ) is designed to hold tight the six supporting rings and thus to define the module geometry. It remains static during all stacking. Before starting the stacking, the six ring elements were placed in position by using the 12 calibrated bars. Then each ring was fastened, at both ends, to the stacking frame.

The stacking was done gap by gap. Before stacking an absorber, all the components except honeycomb spacers were vacuum and alcohol cleaned. As explained in section 3.5 the spacers were cleaned and seal-packed at the production site. The seal was broken only once inside the clean room. Right before stacking, the spacers underwent a high electric field for several minutes. They were rejected if they could not withstand it.

Controls were regularly performed during stacking and cabling. First, the sagging of the last mounted absorber was controlled at four given radii for the outer wheel and at one radius for the inner wheel. Figure 15 shows the typical behavior of this sagging, measured at the middle in depth, as a function of the gap number. A positive value means that the measured point is below its nominal position. A negative value, at the end of the stacking, would correspond to a bulging of the module (never observed in any module). Usually, after ten to twenty gaps, the sagging tends to stabilize around 1 to $2 \mathrm{~mm}$. For the last mounted modules, a predefined positive sagging was applied for the first mounted absorber to reach faster the stabilization.

Once a new gap was stacked, a high voltage test was performed at nominal voltage for several minutes. In case of the occurrence of a trip and its confirmation, the gap was dismounted then re-stacked with new tested honeycombs. The faulty spacers where re-cleaned and tested before reusing them.

Every evening, all the gaps stacked during a day were tested using a low frequency ( 1 and $20 \mathrm{~Hz}$ ) test pulse sent through the high voltage cables and readout back through the signal channels with an oscilloscope as described in detail in [16]. This test (called TBF) checks the integrity of the high voltage buses as well as the signal continuity. Then, these gaps were tested overnight under high voltage. In case of trip or bad low frequency test, the faulty gaps were identified and 


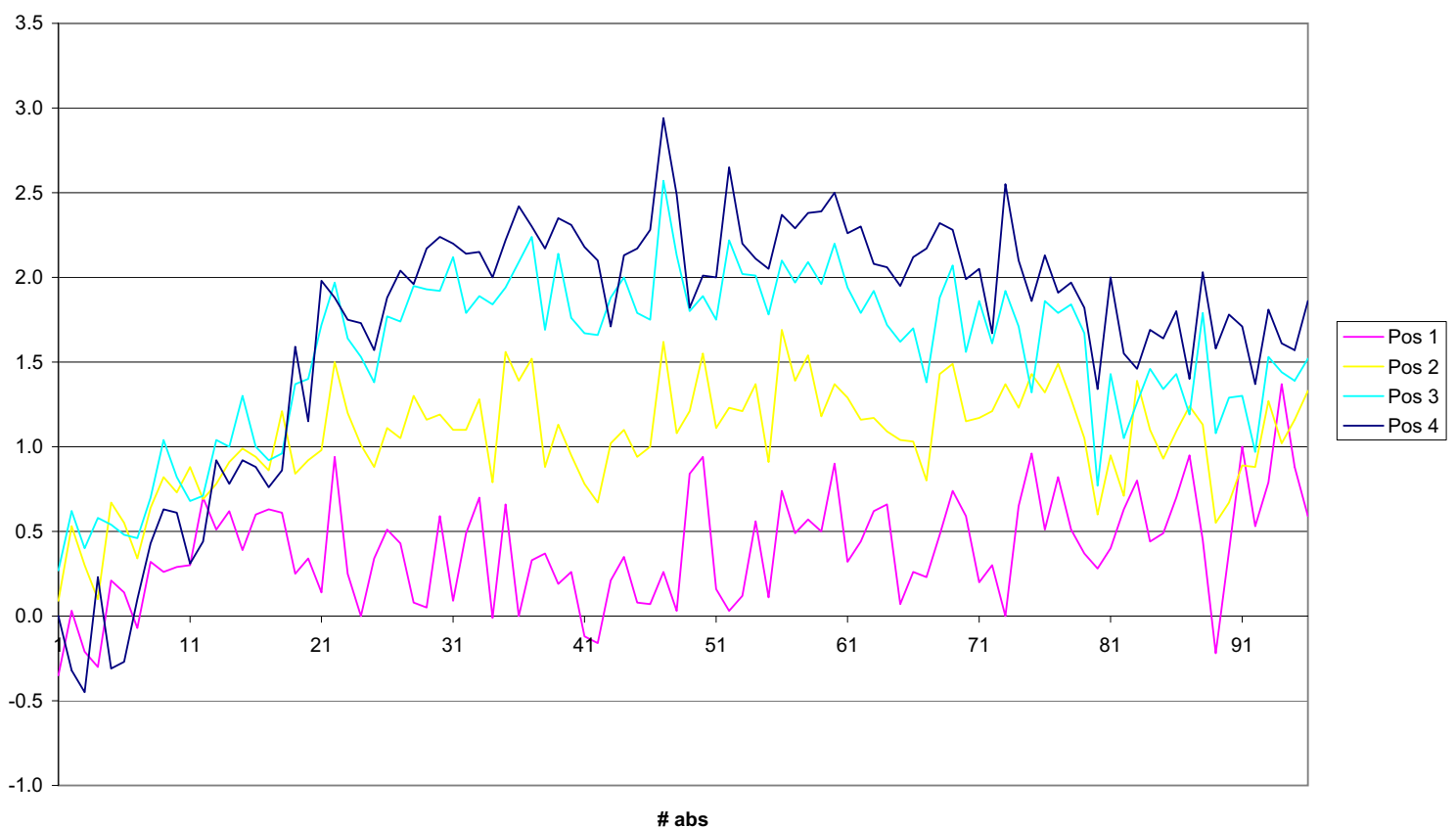

Figure 15. Sagging measurements for a typical module (outer absorbers) as a funtion of the number of stacked gaps. The measurements shown were performed at the middle of the absorber (in depth). Position 1 (4) correspond to the largest (smallest) radius end of the absorber.

unstacked. At the end of each week of stacking, all the stacked gaps were tested over the weekend at nominal high voltage. Again, in case of trip, the faulty gaps were identified and unstacked. Thanks to a positive learning curve and by applying very stringent cleanliness cautions, very few gaps were unstacked for the last modules.

For each module, at the end of stacking, all the gaps had to sustain more than sixty hours under nominal high voltage to be qualified.

After cabling (section 5.4), the geometry of the module was again checked with the calibrated bars and adjusted, mainly by tuning the tension of the longitudinal bars of the large absorbers (section 3.3.1). Then, a frame (hereafter called spatula) was fastened to the back structural rings. This spatula (figure 16) was used to support the module for handling, storage and transportation. Later the module was rotated and laid down in horizontal on its spatula. Before shipping to CERN, the module and the cables were packed together inside a tight plastic bag.

\section{Cold electronics and cabling}

Although the geometries of barrel and end-cap parts of the ATLAS EM calorimeter are different, the designs of the readout and calibration electronics as well as the high voltage distribution have been kept as similar as possible. Hence many technical issues presented in the section 4 of 


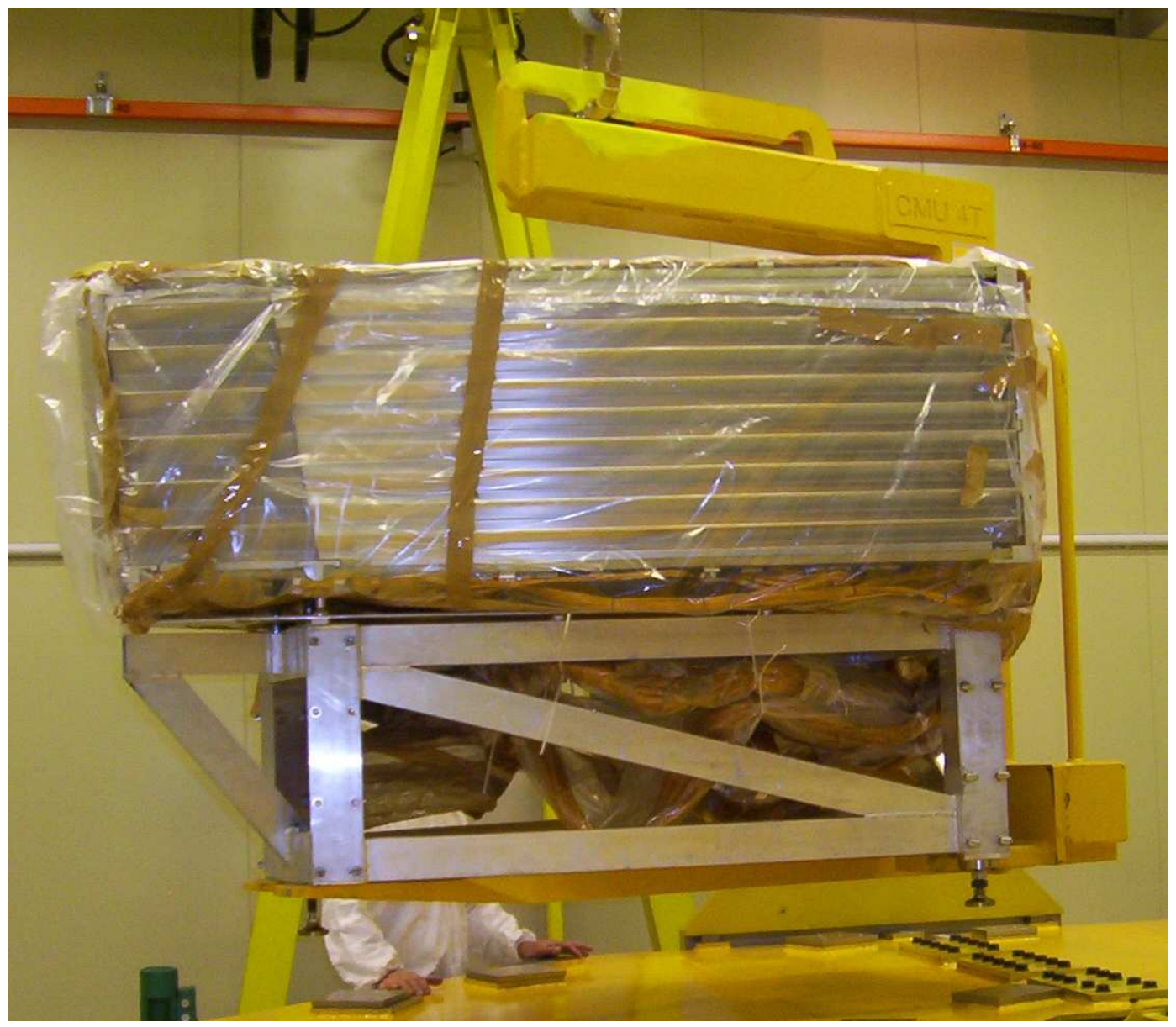

Figure 16. Positioning of a module on the assembly table. Observe the spatula and the lifting frame.

ref. [8] devoted to the EM barrel calorimeter construction apply also to the end-caps. They will be summarized here, emphasing the end-cap specificities.

In order to cope with the $25 \mathrm{~ns}$ interval between bunch crossings of the LHC, a fast shaping of the calorimeter is necessary. For this purpose, the time constant of the CR-RC ${ }^{2}$ shaping amplifier following the preamplifier was chosen to be $15 \mathrm{~ns}$. At this frequency, a cable between a detector cell (a capacitance) and the preamplifier behaves mainly as a transmission line. This means that its capacitance does not contribute significantly to the noise, even for cable lengths of several meters. Taking advantage of this fact, it was decided, for better reliability, to put preamplifiers outside, in front-end crates located on cryostat feedthroughs. Thus, cold electronics comprises only passive components.

\subsection{Summing boards and mother-boards}

The signals coming from electrodes are readout on the front and back sides of the EMEC, by two layers of interconnected boards. While the $\Delta \eta$ granularity is defined by the pattern of the electrode copper pads, summing boards gather the signals from adjacent electrodes generating the $\Delta \phi$ granularity. These summing boards, composing the first layer, are followed by the mother- 
Table 4. mother-board coverage in $\eta$ and $\phi$.

\begin{tabular}{|cccc|}
\hline mother-board type & $\Delta \eta$ & $\Delta \phi$ & number of summing boards \\
\hline F1 & $1.375-1.5$ & 0.2 & 1 \\
F2 & $1.5-1.6$ & 0.2 & 2 \\
F3 & $1.6-1.8$ & 0.2 & 4 \\
F4 & $1.8-2.0$ & 0.2 & 4 \\
F5 & $2.0-2.5$ & 0.2 & 5 \\
B1 & $1.5-1.6$ & 0.2 & 1 \\
B2 & $1.6-1.8$ & 0.2 & 2 \\
B3 & $1.8-2.1$ & 0.2 & 3 \\
B4 & $2.1-2.5$ & 0.2 & 4 \\
IB & $2.5-3.2$ & 0.4 & $2 \times 2$ \\
\hline
\end{tabular}

boards. These mother-boards route the signals to the readout cables and distribute the calibration pulses through accurate resistor networks. Summing boards and mother-boards are electrically separated by thin $\mathrm{G} 10$ boards. In the precision region $(1.5<|\eta|<2.5)$ the electrode connection scheme is similar to the barrel one: the first sampling is readout from the front side, whereas the second and third samplings are readout from the back. In the inner wheel $(2.5<|\eta|<3.2)$ both longitudinal compartments are readout from the back, whereas in the low pseudorapidity region $(|\eta|<1.5)$ they are readout from the front.

In the outer wheel, each summing board services 12 electrodes $(\Delta \phi=0.1)$. In the front section, all 12 electrodes are summed into one readout cell while the sums in the middle and back sections have finer granularity ( $\Delta \phi=0.025$, or 3 adjacent electrodes). The geometry of the end-caps of the EM calorimeter drives to a circular shape for each summing board. In addition, as the distance between adjacent electrodes varies with the radius, 16 different types of summing boards were designed for the front side of the outer wheel (see figure $\emptyset$ ), while 10 types of summing boards were needed on the back side. In the inner wheel, each summing board services 8 electrodes $(\Delta \phi=0.2)$. For both first and second samplings, 4 adjacent electrodes are summed into one readout cell. Only 2 types of summing boards were designed to equip the inner wheel.

The summing boards ${ }^{14}$ are PCBs with low-impedance $15 \Omega$ strip-lines whose lengths are equalized in $\phi$ in order to ensure a low, close to uniform, inductance of $25 \mathrm{nH}$.

Five different types of mother-boards are used on the front side and four types on the back side of the outer wheel. Only one type of mother-board is used on the back side of the inner wheel. All mother-boards have rectangular shapes. The $\Delta \eta \times \Delta \phi$ zone covered by an EMEC mother-board varies with the radius. Table 7 summarizes some characteristics of each type of mother-board. In the outer wheel, 32 mother-boards of each type are needed per end-cap. In the inner wheel, 16 mother-boards are needed per end-cap.

The receptacles at the output of the summing boards and the input of the mother-boards are connected via pins mounted on two thin printed-circuit boards. Each is connected twice to provide

\footnotetext{
${ }^{14}$ The summing boards have been manufactured by ELATE SA, Madrid and TEYDISA, Madrid.
} 
maximum robustness and redundancy. For the 2 nd and 3rd calorimeter layers, every other printedcircuit board also houses a low-capacitance transient voltage suppressor (TVS) [17] with a $6 \mathrm{~V}$ turnon to protect the calibration resistors mounted on the mother-boards against accidental discharges.

The mother-boards route the outputs to the readout cables through "low profile" connectors designed to minimize cross-talk between channels [18, 19].

\subsection{Signal and calibration cables}

The cables used for signal readout, calibration pulse distribution and high voltage powering are identical to those used for the EM barrel calorimeter. The characteristics of these cables, like their design, manufacture and tests, are described in section 4 of ref. [8].

Two groups of cable impedance and preamplifier were used in accordance with detector cell capacitances. $50 \Omega$ cables were connected to low capacitance cells i.e the presampler and calorimeter strip section in the precision region $(1.5<|\eta|<2.5)$. Other calorimeter cells (middle and back sections of the precision region, both longitudinal compartments of the inner wheel and the $1.375<|\eta|<1.5$ region) were readout through $25 \Omega$ cables. Cables of $50 \Omega$ impedance are also used to carry calibration pulses to the mother-boards. The lengths range between 3.2 and $5.4 \mathrm{~m}$ for signal cables, 4.9 and $6.0 \mathrm{~m}$ for the calibration cables. The same packaging (i.e harnesses of 64 mini $\mathrm{Cu}-\mathrm{Ag}$ coaxial cables, low profile connectors and micro-D connectors ${ }^{15}$ ) as for EMB was chosen. EMEC cables were produced in parallel with the EMB ones by the same manufacturer. ${ }^{16}$

\subsection{High voltage boards and cables}

A complete description of the EMEC high voltage cabling can be found in ref. [20]. As explained in section 2, the high voltage setting changes by steps with $\eta$ to partially compensate the liquid argon gap variation. As shown in figure 12, the outer (inner) wheel is divided into seven (two) high voltage sectors. Furthermore, in the outer wheel, electrodes are supplied by groups of 24 $(\Delta \phi=0.2)$, connected in dedicated HV boards. As at high rapidity currents drawn by minimum bias events are larger, electrodes in the inner wheel are grouped by 4 only. In this case, each HV board supplies 4 groups (i.e. 16 electrodes, $\Delta \phi=0.1$ ). Each electrode being surrounded by two gaps, high voltages are distributed on both electrode sides named HV1 and HV2. ${ }^{17}$ For better reliability HV1 and HV2 sides are fed by two different power supply channels. In one EM end-cap calorimeter, the high voltage is then distributed on 704 independent sectors, defined by $7 \times 32$ $(2 \times 64) \eta \times \phi$ sectors and $2 \mathrm{HV}$ sides for the outer (inner) wheel.

In order to avoid possible HV discharges or Corona effects in coaxial cables, single conductor, Polyether-ether-ketone (PEEK) insulated cables were selected for HV connections. HV cables were grouped in harnesses of 8. Each HV cable was soldered to the HV boards at the module level. At the patch panel level, an 8-point custom developed ATI connector ${ }^{18}$ was chosen.

\footnotetext{
${ }^{15}$ Micro-D twistpin connectors from ITT/Cannon.

${ }^{16}$ Axon cable SA.

${ }^{17}$ The high voltage side called HV1 (2) belongs to the single (double) sided laminate used to produce the electrodes (see section 3.4). The dissymetry of the insulating layers reflects directly in the blocking capacitances between the signal layer and both HV sides.

${ }^{18}$ ATI Technologies Inc.
} 


\subsection{Cabling procedure}

After completion of the stacking of a module, the summing boards (figure $\rrbracket$ ) and the HV boards were plugged into electrode connectors. Before their installation, electric continuity tests of the summing and HV boards were performed to ensure that neither signal nor HV channel was accidently grounded. The connexion of the summing boards was checked, first visually, then with a quick capacitance test with the help of an RLC-meter. Then the HV cables were soldered on the boards. Thin G10 boards, completely covering the summing and HV boards, were installed. Their aim was to electrically isolate mother-boards from summing boards and to protect the cables from contact to the numerous solderings on the summing boards. The mother-boards were installed by plugging pin carriers or TVS PC-boards going through the mother-boards to the summing boards, via holes bored in the insulating sheets. The cable harnesses for signal readout and calibration were installed, the low profile connectors plugged into the mother boards at one end, the other end being mounted on metallic patch panels. These ones were fixed at the end of provisional rails, fastened to the stacking frame. The aim of these rails was to mimic, in length and location, the trays devoted to house the cables along the cold wall of the cryostat. A limited space has been defined for the cables in order to let a free gap between the envelop of the electromagnetic calorimeter and the envelops of the cryostat and of the hadronic calorimeter. To keep all the cables inside this space, they were carefully routed then, on top of them, composite bars were screwed to the module structure.

\subsection{Monitoring instruments}

As the drift veloticity, and thus the signal, depends on liquid argon temperature, this one is continuously monitored by a system [21] similar to the EM barrel one (section 5 of ref. [8]). In each EM end-cap calorimeter, 48 calibrated $(\Delta \mathrm{T}=10 \mathrm{mK}) \mathrm{PT} 100$ platinum resistors have been glued on absorbers along the front and back longitudinal bars. In the outer wheel, 32 of these temperature probes were located at a radius of $193 \mathrm{~cm}$, for both front and back sides. In the inner wheel, the 16 other probes were glued at $\mathrm{R}=52(61) \mathrm{cm}$ on the front (back) sides. In both wheels, these probes are uniformly distributed in $\phi$.

Electronegative impurities can also affect the collected charge and degrade the performances of the calorimeter. In order to control the liquid argon purity, 10 purity monitors have been installed in each end-cap cryostat. Two of them are mounted on the outer radius of the EM calorimeter, within the wheel structure close to the vertical plane, one at the top and the other at the bottom. Each device contains two radioactive sources (an ${ }^{241} \mathrm{Am}$ source emitting $5.5 \mathrm{MeV} \alpha$-particles and a ${ }^{207} \mathrm{Bi}$ source emitting $1 \mathrm{MeV}$ conversion electrons) whose ionization charges are collected within two separated liquid argon gaps. The ratio of these measured charges allows to extract the absolute oxygen content in liquid argon. Relative changes can be measured with a precision better than $10 \mathrm{ppb}_{2}$-equivalent contamination. More details about the purity monitors can be found in section 5 of ref. [8] and in ref. [22].

\section{Cold and beam tests}

All EMEC modules were tested for qualification in liquid argon at CERN. The main goals were to verify the high voltage holding, to check the behavior of the readout cells and to measure their 
characteristics. A complete cold test sequence lasted about 1 month per module. These tests extended over approximately three years, from September 2001 to April 2004. Three modules were also tested under electron beams. Each cold test, like each beam test, was preceded and followed by warm tests.

\subsection{Experimental set-up}

In order to save money, the cryostat of the former NA31 experiment [23] was recovered and suitably modified to accommodate three signal feedthroughs (associated to ATLAS-like front end crates) and a high voltage feedthrough. This HV feedthrough did not comprise enough channels. As the fine $\phi$ granularity of the HV distribution in the inner wheel is driven only by the minimum bias events at LHC, during these tests the HV lines of the inner wheel were grouped by four (i.e. 16 electrodes).

The cryostat was connected to a common cryogenic system [24] used by all the ATLAS liquid argon cold and beam tests in the North Area beam lines H6 (EMEC, HEC and FCAL modules) and H8 (EMB modules). The cool-down was performed with liquid nitrogen circulating in a cooling circuit, and the warm-up with electrical heaters. At a rate of $2 \mathrm{~K} / \mathrm{h}$, each operation lasted about 5 days. The purity of liquid argon was measured with an ATLAS-type purity monitor (see section 5.5.

Beam tests were carried out at the CERN's SPS H6 beam line, using electron and positron beams with energies from 10 to $150 \mathrm{GeV}$. The beam contained also muons, vetoed at the trigger level, and about $10 \%$ of pions, tagged and removed offline using scintillator counters located behind the detector. The beam line was instrumented with four proportional chambers [25] to reconstruct the particle path. The dead material thickness in front of the active part of the calorimeter (cryostat walls, cold electronics, cables, liquid argon, ...) was almost constant with $\eta$ and estimated to $1.5 \pm 0.1 X_{0}$.

\subsection{High voltage tests}

Before insertion of the module into the cryostat, an HV test was performed at half of the nominal high voltage values. This allowed to identify potential problems such as short circuits or disconnected HV channels. Voltage ramp up was done at $11 \mathrm{~V} / \mathrm{s}$. The sensitivity of the current measurement was good enough to see the dissymetry between the two HV sides (see sections 3.4 and 5.3).

After filling up the cryostat with liquid argon, the test was carried out at nominal high voltage. During a standard test, the module remained under voltage about one week. In case of a beam test, the high voltage was kept for three to four weeks.

After warming-up, the detected problems were investigated and cured in a clean room. Each faulty electrode was isolated and connected to a spare HV cable. When a short was found in liquid argon but not visible at room temperature the faulty HV sector was split into two halves, one being connected to a spare cable. After completion of these operations, a last HV test was performed before the storage of the module.

\subsection{Signal and calibration test}

A signal and calibration test (called TPA) performed a crude calibration of each readout cell. Its goal was not to extract accurate calibration constants, but to perform a complete check of the 
a
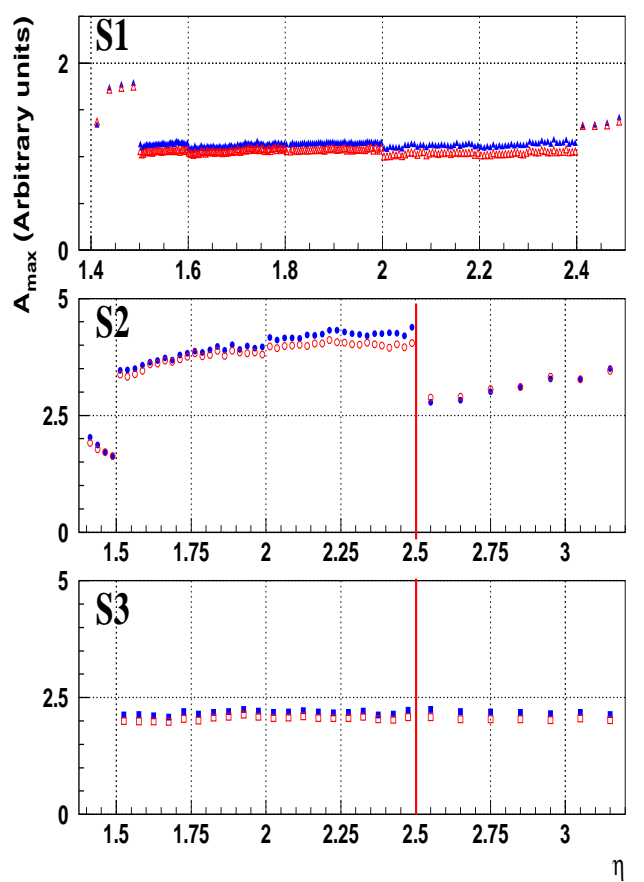

b
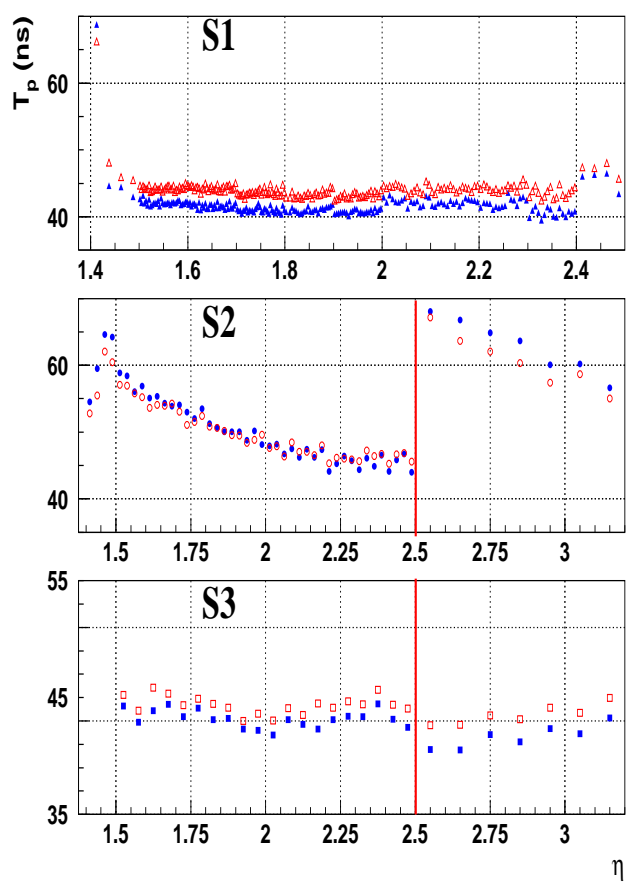

Figure 17. $A_{\max }$ (a) and $t_{p}$ (b) as a function of $\eta$ for the front, middle and back samplings, at cold (solid symbols) and room (open symbols) temperatures.

electrical continuity, of the cabling quality and more generally of the correct behavior of the readout cell response.

Each calibration line was pulsed with a $20 \mathrm{~V}$ amplitude and $2 \mathrm{~ns}$ rise time step signal. The responses of all cells connected to this calibration line were multiplexed and read out by a digital oscilloscope with an automatic procedure, averaging 50 pulses to reduce noise. The average signals were recorded and analyzed on a PC.

A software shaping, modeling the CR-RC ${ }^{2}$ filter used in the front end electronics, was then performed. This step was mandatory to compare results with the ones produced with the ATLAS readout electronics and to validate the testing procedure [16]. Maximal amplitude $A_{\max }$ and rise time $t_{p}$ were then computed for each channel. Figure 17 shows typical $A_{\max }$ and $t_{p}$ values for the three samplings as a function of $\eta$, at room and cold temperatures. Expected module geometrical effects can be observed for example at $\eta=1.5$ or 2.5. A correlation, linked to cell capacitance variations, is expected between $A_{\max }$ and $t_{p}$. It can clearly be seen in the middle sampling.

The sensitivity of this test to the cell capacitance was around $100 \mathrm{pF}$ allowing to detect problematic channels by non expected $A_{\max }$ and/or $t_{p}$ values. Singular channels were divided in four categories:

- dead channel (cut signal or calibration cable, short circuit between ground and signal, ...);

- channel with low $A_{\max }$ and high $t_{p}$ linked to a higher capacitance than expected (i.e. HV problem, high capacitance on the electrode or on the cold electronics); 
- channel with high $A_{\max }$ and low $t_{p}$ due to a capacitance lower than expected (1 electrode unplugged on the summing board, no or badly plugged pin between mother and summing boards);

- channel with high $A_{\max }$ and $t_{p}$ linked to the absence of the ground return on the signal cable.

These problems were cured whenever possible. Cabling mistakes were also identified and corrected for.

\subsection{Beam test results}

Along the production, three modules were exposed to electron beam in the H6 beam line of the CERN experimental North Area with ATLAS-like electronics in similar conditions as the full-size pre-production module, whose performance were reported in [9]. The main purposes of these tests were to check the electron response reproducibility between modules and to control the production quality by measuring the detector response uniformity over its whole acceptance. The nonuniformity of the EMEC modules comes from the stepped high voltage setting (figure 6 ), the energy leakage outside the cluster and the accordion geometry. All these effects can be parametrised in pseudorapidity over the whole calorimeter acceptance. It has been found that the same values of the parameters can be applied to the three modules. These parametrizations can therefore be used at the LHC start and to tune the simulation. An unexpected non-uniformity in $\phi$ coming from local fluctuations of the gap thicknesses was observed [26] and corrected for with the $\mathrm{S} 2$ cell capacitance measurement performed on each module (see section 8.4.3).

The energy resolution in each cell can be parametrized, after noise subtraction, with the expression:

$$
\frac{\sigma_{E}}{E}=\frac{a}{\sqrt{E}} \oplus b
$$

where $a$ is the sampling term, and $b$ the local constant term reflecting local non-uniformities in the calorimeter response. The energy $E$ is expressed in $\mathrm{GeV}$. A sampling term between $10 \% \mathrm{GeV}^{1 / 2}$ and $12.5 \% \mathrm{GeV}^{1 / 2}$, depending on $\eta$, and a local constant term between $0.3 \%$ and $0.4 \%$ have been measured. The global constant term of the energy resolution combines the local constant term and the response non-uniformity from cell to cell. The later can arise from mechanical, geometrical, calibration and material effects. To assess it, each whole module has been scanned using a $119 \mathrm{GeV}$ projective beam. The non-uniformity is then measured as the dispersion (r.m.s./mean) of the average energies measured in every cell. Excluding only cells close to the edges and those close to the crack region between outer and inner wheels, it is found around or below $0.6 \%$ for the three tested modules.

The global constant term can be estimated from the overall energy spectrum shown in figure 18. More than 16 millions electrons enter this distribution, collected in 2455 cells of the three modules kept in the analysis, representing an area of $\sim 3 \mathrm{~m}^{2}$. Results from the three modules have been combined without any energy scale normalisation between modules. The low-energy tail is mainly due to material effects upstream the cryostat and residual pion contamination of the electron beam. The global constant term is estimated from a Gaussian fit to the spectrum over the region not affected by the tail (starting at $-1.5 \sigma$ of the mean value), after unfolding the average stochastic term $(0.114 / \sqrt{119}=1.05 \%)$, the noise term contribution $(200 \mathrm{MeV} / 119 \mathrm{GeV} \sim 0.17 \%)$ and 


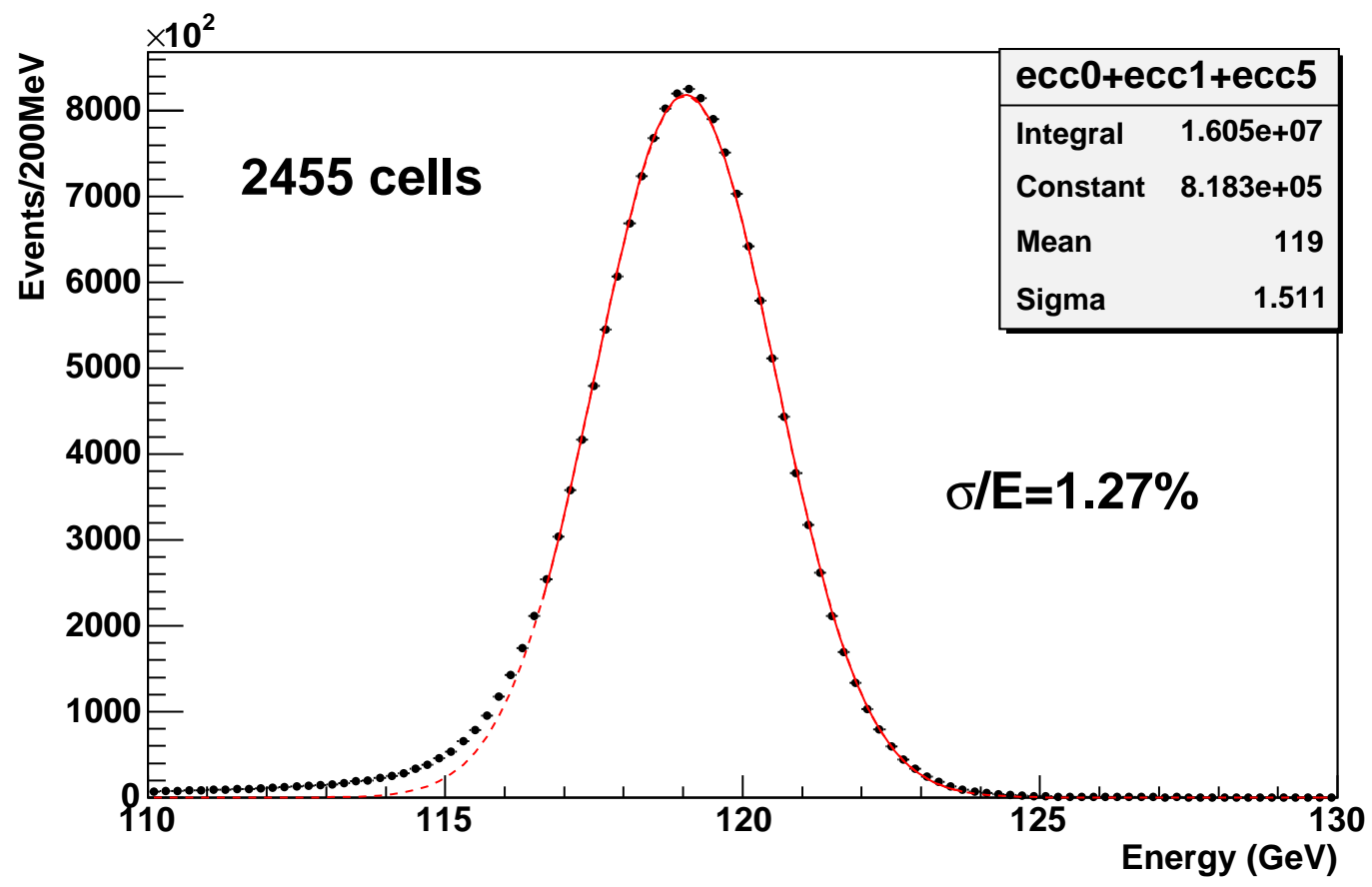

Figure 18. Overall energy spectrum of $119 \mathrm{GeV}$ electrons collected in the three modules, over 2455 cells covering an area of $\sim 3 \mathrm{~m}^{2}$. The result of a Gaussian fit starting at $-1.5 \sigma$ off the mean value is shown.

the beam momentum spread $(\sim 0.07 \%)$. A value of $0.70 \pm 0.04 \%$ is found, consistent with the quadratic sum of the local constant term and of the detector response non-uniformity. All the measured performances meet the challenging physics requirements despite the complex geometry of the detector. More details can be found in [26] and [11] .

\section{End-cap cryostat}

\subsection{Cryostat characteristics}

Each cryostat (figure 19] [27] houses three calorimeters: the electromagnetic and hadronic endcaps (EMEC and HEC [28]) and the forward calorimeter (FCAL [29]). A cryostat is composed of two vessels made of aluminium 5083. The cold vessel is filled with liquid argon. The warm vessel is loaded by the external atmospheric pressure. The space in between the vessels is evacuated to at least $10^{-2} \mathrm{~Pa}$ and contains multilayer super insulation. The cold vessel (inner volume diameter $4.3 \mathrm{~m}$, length $2.8 \mathrm{~m}$ ) supports 219 tons of detectors and 26 tons of liquid argon filling.

The cryostat vessels form a cylindrical torus around the beam axis with a flat wall in front (towards the interaction point) and a removable cover plate in the back (away from the interaction point). All cryogenic and electrical feedthroughs are integrated onto the back-end, which as a result extends to a larger cylindrical radius. The outer warm vessel of the cryostat is embedded in the tile calorimeter, which provides four support bearings (two at each end) slightly below the middle plane. 


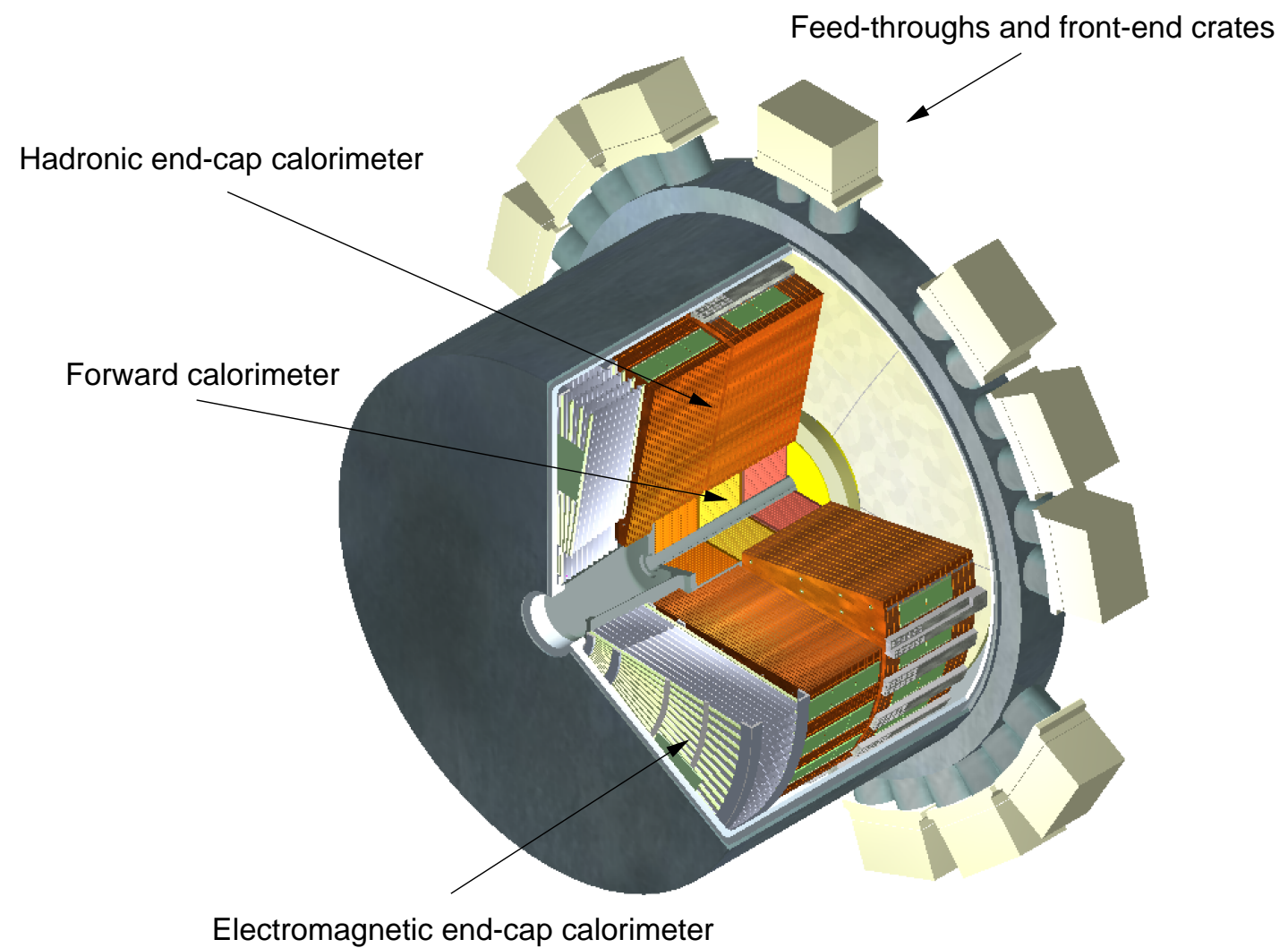

Figure 19. Cut-away view of an end-cap cryostat showing the position of three end-cap calorimeters: EMEC (first wheel with accordion and its presampler in front), hadronic (two wheels) and forward (3 cylinders at small radius) calorimeters. Also visible the signal feedthroughs with their associated front-end crates (two feedthroughs per FEC).

The inner warm tube provides space for the beam pipe and an associated pump station. It is bolted to the warm front wall. It is fixed to the warm cover plate at its back end via a flexible connection. The cold inner tube surrounds the inner warm tube. The back part of it is a double tube, the FCAL structure, housing the FCAL detector. The smaller radius tube forms the wall of the cold vessel along the $z$ axis. The larger tube supports the FCAL detector and connects rigidly the cold front wall to the back cover plate, thus providing mechanical stiffness to the cold vessel.

The warm front wall is flexible ( $15 \mathrm{~mm}$ thick) and, under operating conditions, sits on the cold front walls through insulating spacers. The cold front wall is supported through the FCAL structure by the cold back cover ( $88 \mathrm{~mm}$ thick) and may thus keep a reasonable thickness ( $32 \mathrm{~mm}$ ).

Four insulating bearings support the cold vessel within the warm vessel. They are placed such that the forces are transmitted vertically to the supports of the cryostat on the tile calorimeter to avoid geometrical deformation of the warm vessel under loads. A set of insulating stops, in addition to these supports, centers the cold vessel into the warm vessel after a thermal cycle. Rails fixed along the inner wall of the cold vessel slightly below the median plane on both sides support the EMEC and HEC detectors. The lateral position is provided by a guiding rail fixed at the top of the cold vessel. The longitudinal position of the detectors is given at their back end by stoppers. 


\subsection{Feedthroughs characteristics}

For each end-cap cryostat, 25 signal feedthroughs bring 48000 signal, calibration, monitoring and spare lines through an insulating vacuum, out of the liquid argon environment, into the warm one. They are radially distributed at the end close to the covers. Twenty of these feedthroughs are fully devoted to the EM calorimeter. A few EMEC channels are also connected via the four feedthroughs mainly used for the hadronic end-cap calorimeter. The last feedthrough is devoted to the forward calorimeter.

The signal feedthroughs have been designed to minimize heat conduction, to preserve the insulating vacuum of the cryostat and to accomodate the relative movements of the warm and cold walls during cooldown and warmup. They have also to minimize the cross-talk between lines and to ensure an excellent ground return.

A signal feedthrough consists of a warm flange and a cold flange, with a flexible bellows welded between them. Each flange houses four pin carriers with a total of 30 insulating glass rows, with 64 gold-plated pins per row, providing a total of 1920 connections per feedthrough. Both flanges are interconnected with flat, polyimide strip-line flexible $33 \Omega$ cables. This choice allows a single type to be used for both the 25 and $50 \Omega$ cables. More details on the design, the construction and the tests of signal feedthroughs can be found in ref. [30].

At the same end as the signal feedthroughs, two HV ports are mounted close to the highest point of each end-cap cryostat. Each port holds $840 \mathrm{HV}$ lines, divided over four wire feedthroughs. One of these ports is devoted to the EMEC detector. Each port consists of a single warm bulkhead erected on the warm vessel from which the wire bundle leads to the calorimeter via a tube connected to the cold vessel by stainless steel bellows. The liquid rises up to the bellows followed by a 70-cmhigh column of argon gas. Warm O-rings and RF gaskets between the ports and the warm vessel ensure gas tightness and close the Faraday cage at the level of HV filters mounted on the top of the HV feedthrough port. The HV wire consists of a solid $0.41 \mathrm{~mm}$ diameter constantan conductor with a 0.30 -mm-thick PEEK cladding. ${ }^{19}$ All wires are stripped inside the wire feedthroughs and potted, insuring a full wire continuity between the warm connectors ${ }^{20}$ and the cold connectors on the patch panels. More details on the design, the construction and the tests of high voltage feedthroughs can be found in section 6.3 of [8] and in ref. [31].

\section{Integration}

\subsection{EMEC wheel assembly}

Each EM end-cap calorimeter consists of 8 modules. The wheel assembly took place in a ISO 8 class clean room at CERN, where both temperature and humidity were controlled. The first wheel (called ECC or C wheel) was assembled and integrated in spring and summer 2003, while the second wheel (ECA or A wheel) was completed in summer 2004.

A dedicated stand was developed, which was used both for the wheel assembly in horizontal position and for the rotation of the calorimeter to vertical position (see section 8.3). The table is made of two halves (figure 20), one of them being able to slide horizontally.

\footnotetext{
${ }^{19}$ Habia Cable, Söderfors, Sweden.

${ }^{20}$ REDEL, Kft S-series 51-channel 3 kV connectors, model SLA.H51.LLZG, Lemo SA, Switzerland.
} 


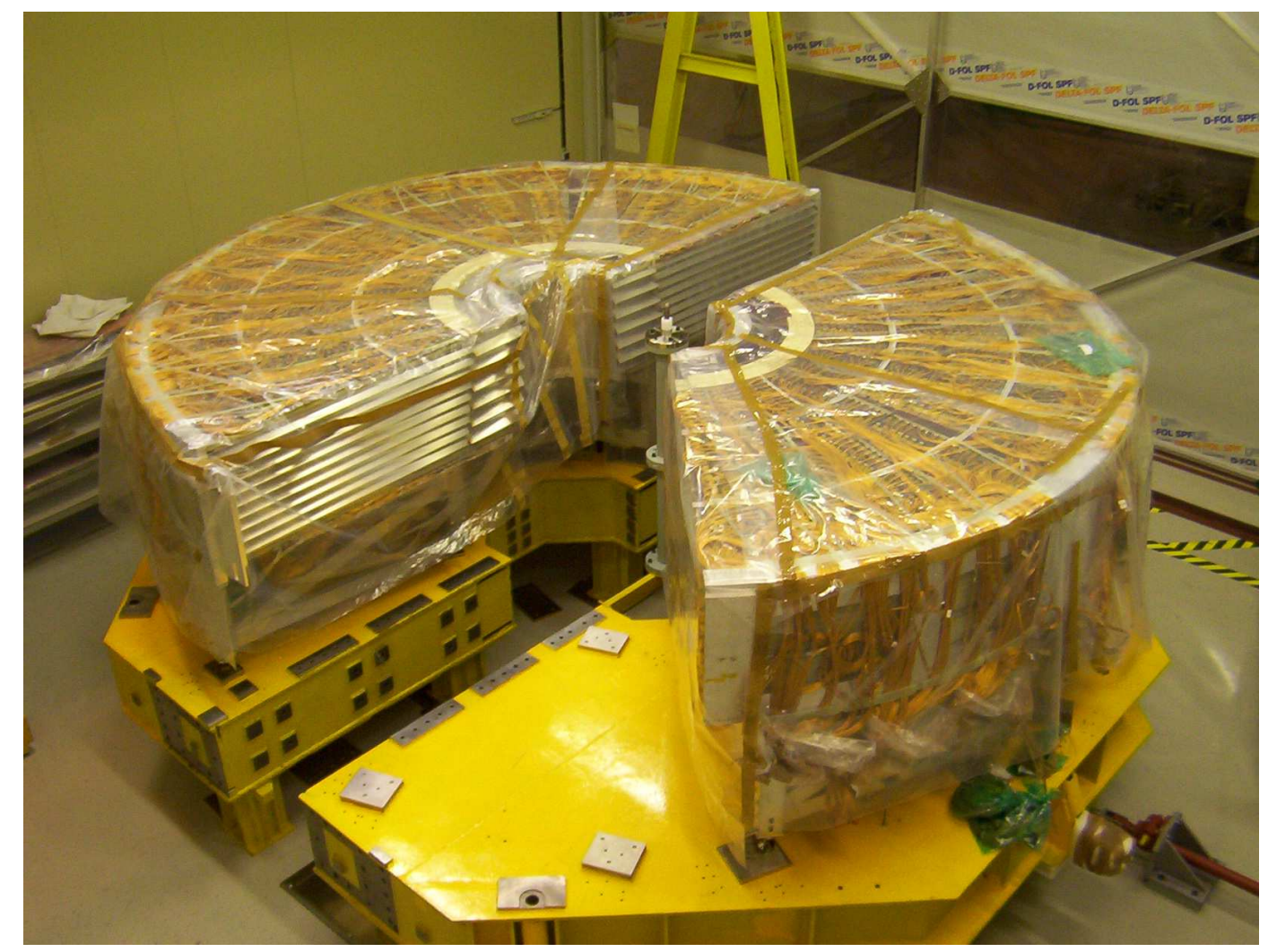

Figure 20. This picture shows the assembly table opened just before the installation of the last module. Observe the three reference plates on which the feet of the spatula of this last module will lay. The central axis is also visible.

To avoid the precision machining of the whole table, 24 flat stainless steel plates were accurately adjusted on the upper side, to define the reference horizontal plane. A central axis defined the centre of the wheel and two dummy rails, mounted on two lateral arms fastened on the table, gave the position of the cryostat rails. The geometry of the whole device has been surveyed prior to wheel assembly.

The process of the calorimeter assembly was the same for every module (resting on its spatula). The first step, consisted of a high voltage test (see section 8.2). Then a mechanical adjustment was performed in order to correct any module deformations. These deformations occurred during the cold tests, when the modules were not sustained by their spatula. A dedicated device was developed to correct the module shape. The shape was checked using the calibrated bars. Thanks to this correction, the mean deviation with respect to the nominal geometry has been decreased to 0.19 $\mathrm{mm}$ for the front face and to $0.07 \mathrm{~mm}$ for the rear face. The difference between front and rear faces comes from the rigidity of the frame that holds the module during the transport and the handling operations. Once the correction of the geometry is done, a new high voltage test is performed.

The last gap was carefully cleaned and equipped with two electrodes, one for the outer wheel and one for the inner wheel. Because these electrodes had to be installed in vertical position, they had the spacer nets glued on them by a few dots. Then, the module and the spatula were lifted using a dedicated lifting tool (figure 16) and moved to the table with a crane installed inside the 
Table 5. Survey of both EMEC wheels on the assembly table (horizontal). All measurements concern the front rings and are given in $\mathrm{mm}$. The nominal radii are given as reference. Coordinates $(\mathrm{x}, \mathrm{y})$ of the centres of the intermediate and inner rings are given with respect to the measured center of the outer ring. The accuracy is estimated to $0.3 \mathrm{~mm}$.

\begin{tabular}{|lccccc|}
\hline & Nominal & \multicolumn{2}{c}{ A wheel } & \multicolumn{2}{c|}{ C wheel } \\
& Radius & Radius & Centre & Radius & Centre \\
\hline Outer ring & 2064.0 & 2064.0 & 0,0 & 2063.8 & 0,0 \\
Intermediate ring & 661.0 & 661.3 & $0.1,0.1$ & 661.0 & $-0.1,0.2$ \\
Inner ring & 376.0 & 376.4 & $0.5,0.0$ & 376.6 & $0.0,0.2$ \\
\hline
\end{tabular}

clean room. The module was carefully unloaded on the assembly table, the three feet of the spatula laying on the small stainless steel plates. During this first approach, the module was located at a few centimeters of the adjacent one. Actually, the feet of the spatula were equipped with Permaglide plates, with a friction factor of a few tenths in our working conditions. Hence it was possible to move the module to its final position with a moderate force (less than $3000 \mathrm{~N}$ ) applied to the spatula by mechanical jacks. Because of the shape of the waves of the absorbers, the position of the module had to be tuned all along the approach phase. This adjustment was done with respect to the central axis of the assembly stand: the outer ring of the module was kept at the right radius, within $\pm 1 \mathrm{~mm}$, during the whole movement of the module.

When the module was roughly in its final position (few tenths of millimeter), the adjustment was finalized using the junction parts. Six accurate junction parts link a module to the previous one. The positioning was reached when all junction parts could be placed together on both modules. Then, the two modules were fastened together, the gap at the junction being the same than all the other ones. Just after the mechanical positioning of a module, a high voltage test was performed in order to check the behavior of this inter-module gap.

The assembly stand was opened in order to get enough space for the installation of the last module (figure 20). The mobile half was translated by $600 \mathrm{~mm}$, pulled by two jacks while slipping on stainless steel plates fixed on the floor. The feet of this half-table were equipped with Permaglide layers. Once the last module was positioned and the high voltage test performed, the assembly table was closed. It was a delicate operation because, at the end, two gaps had to be fitted simultaneously: between the first and the last modules on one side and the fourth and the fifth modules on the other side. An accurate positioning of the table at the end of its translation was ensured by dowel pins. Hence, as the relative position of the two half-tables was unchanged after closing, the adjustment between the fourth and fifth modules, already tuned before the opening, was done first. Then, the last module was linked to the first one. Finally, the high voltage test was performed on these two last gaps.

The last step of the assembly process was a geometrical measurement of the EMEC wheel with theodolites. The 3 structural rings of the front face have been measured, at 16 points on each ring. The main results are summarized in table 5 .

After this geometrical survey, the completed wheel was ready for its integration inside the cryostat. 


\subsection{High voltage tests during wheel assembly}

As explained in the previous section, during the wheel assembly several high voltage tests were performed at the following steps:

- module reception in the assembly clean room;

- after reshaping of a module;

- after the junction of two modules.

These high voltage tests were performed in air at half of the nominal values, with a ramp up set to $4 \mathrm{~V} / \mathrm{s}$. Ten minutes after reaching the nominal voltage, the leakage current had to be less than $70 \mathrm{nA}$ to accept the corresponding channel.

The main goal of these tests was to prepare a map of the problematic high voltage channels before the integration step. These channels, which were not accessible during the assembly stage, ${ }^{21}$ were investigated during the integration step, while the wheel was in vertical position and the spatulas removed.

As on one edge of a module, before assembly with its neighbour, one electrode was missing in each HV sector, it was possible to calibrate the sensitivity of the loading currents to the number of connected electrodes. As an HV sector gathers 24 (4) electrodes in the outer (inner) wheel, these loading currents should be lower by $4 \%(25 \%)$ when an electrode is missing. Our set-up was sensitive to such differences, thus we were able to identify a bad connection. This method was applied for the first EM end-cap calorimeter during its assembly at CERN and then applied at stacking sites for the eight last modules.

\subsection{Electromagnetic calorimeter insertion into the cryostat}

The end-cap cryostat was placed in a clean room close to the EMEC assembly one, separated only by a large curtain. The EMEC wheel was moved from the assembly room to the integration one by using rollers and an electric trolley, able to pull the load of both the assembly table and the wheel itself. The wheel (still in horizontal position) was positioned close to the cryostat with both cylindrical symmetry axes of the wheel and the cryostat in the same vertical plane.

Then, the table was lifted $5 \mathrm{~m}$ up, using a 60 tons crane and a dedicated lifting beam. A rotator $^{22}$ [28] was positioned below the table, which was unloaded on it (see figure 21). This rotator was equipped with two bearings defining the rotation axis and an electrical jack as rotation engine. The whole system (rotator, assembly table and calorimeter wheel, i.e. about 70 tons) was laying on air-cushion pads, allowing easy and smooth movements.

Once vertical, a survey has measured a maximum deformation of the calorimeter of $2.0 \mathrm{~mm}$ (occurring in the upper part of the wheel) and a very tiny mean radius variation of about $0.1 \mathrm{~mm}$. Both results are in good agreement with our previous finite element model analysis.

The insertion was done using a cradle (hereafter called T6), equipped with two rails (covered with Permaglide plates), aligned and connected with those of the cryostat. To put the wheel on T6,

\footnotetext{
${ }^{21}$ Most of the HV boards are located on the back side of the modules, thus unaccessible when the wheel is in horizontal position.

${ }^{22}$ Designed and supplied by TRIUMF, Canada
} 


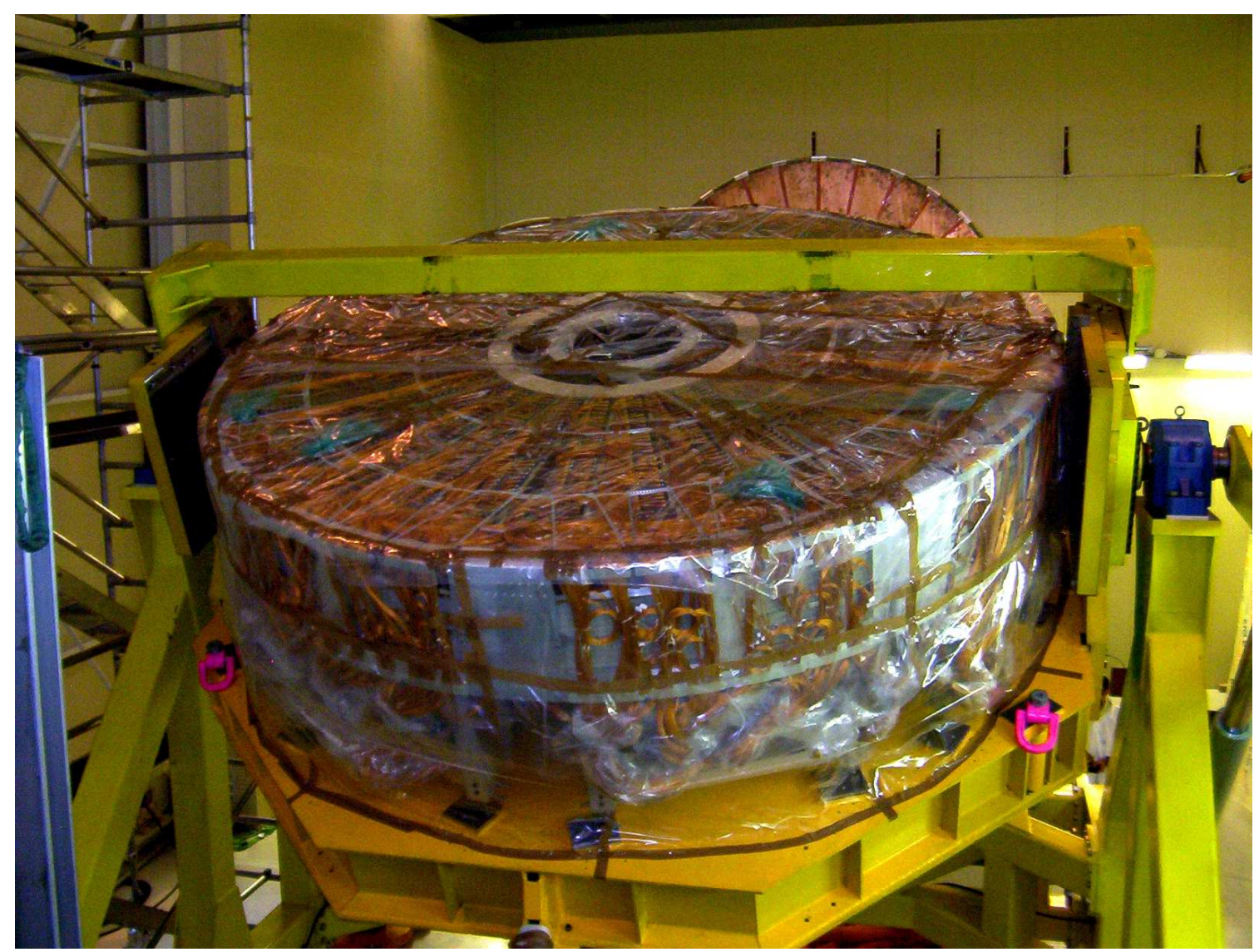

Figure 21. Rotation of an EMEC wheel. The calorimeter lays on the assembly table which is mounted on the rotator. One of the two bearings (in blue at right) is visible.

the rotator was slightly lifted by the air pads. Once the wheel was well aligned, its weight was gradually transferred to T6, by slowly deflating the air-cushion pads. Parts of the assembly table arms were then fastened to T6 and unbolted from the table. Rotator and assembly table were then removed. At this point the first high voltage test of the full wheel in vertical position was carried out (see section 8.4.

Later, the spatulas were removed. A survey of the back side confirmed the measurements of the front one made on the rotator. Because of their weight, the cables were provisionally tied to specially designed structures (figure 22) linked to the outer back ring.

As two neighboring modules share some patch pannels, it was necessary to re-route some harnesses. Before losing access to the front face, a special care was taken to this final cable routing with respect to the front and edges envelopes of the detector. Then a TPA test (see section 6.3) was performed on the front cells, while they were still accessible. Few problems were found (ground disconnections or broken pins in low profile conectors, damaged mother-boards, etc..) and solved.

After this test, the wheel was pushed close to the cryostat, using a push/pull device bolted to both T6 and the EMEC wheel. Before insertion, the wheel had to be aligned on the axis of the cold vessel of the cryostat. This was obtained by aligning the key located at the top of the wheel with respect to a slot inside the cryostat. The positions of the wheel, the top key, the slot and the cold 


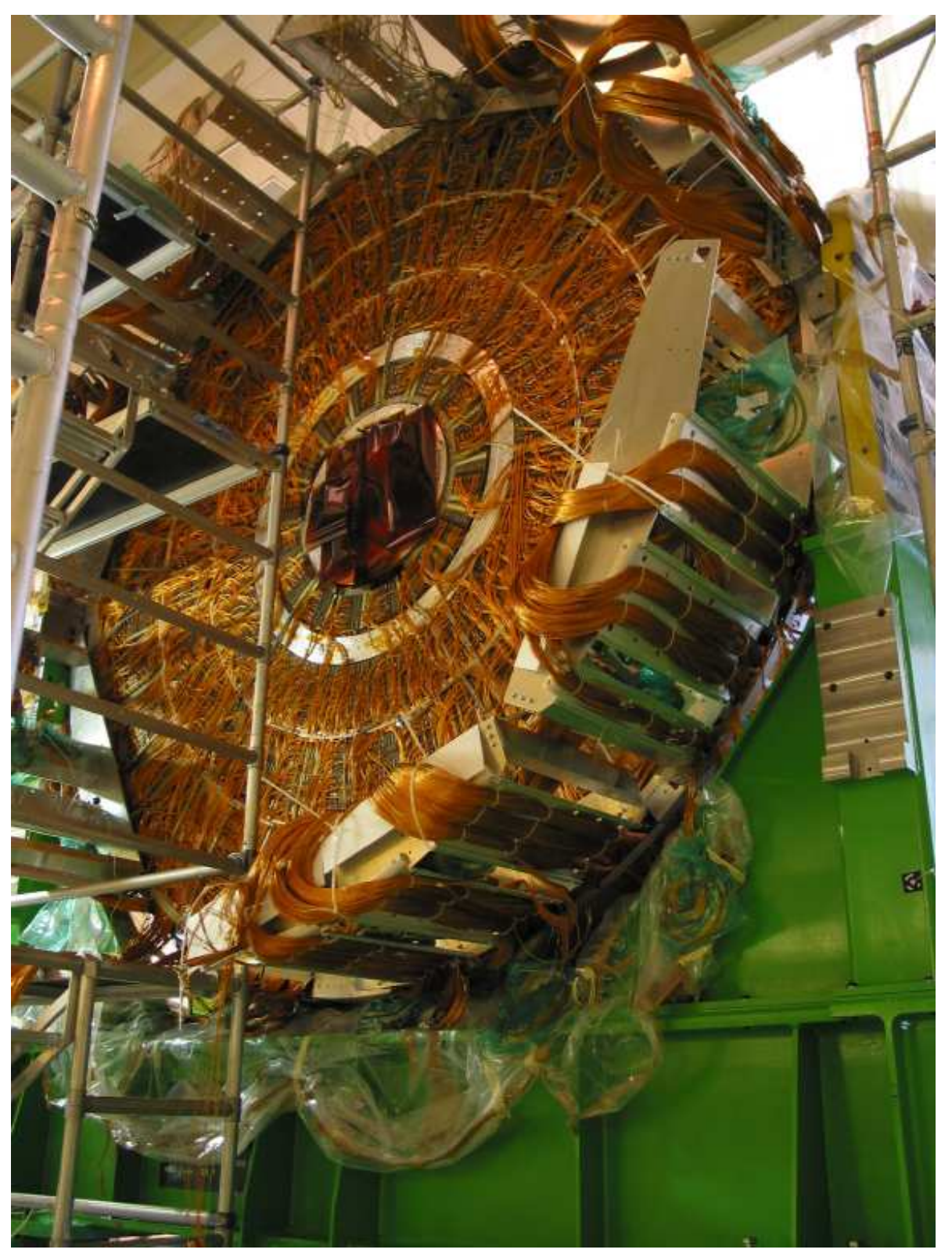

Figure 22. Photo showing the back face of the wheel with the special tools to bring the cable trays at their virtual position and the cables partially routed inside them.

vessel axis have been measured. These measurements led us to a small correction of the top key shape ( $0.1 \mathrm{~mm}$ for the first wheel and $0.8 \mathrm{~mm}$ for the second one). Later, the key and the slot were aligned by pushing the wheel laterally.

The presampler calibration cables share some EMEC calibration harnesses. For that reason during its fabrication and its integration the presampler was not connected to its calibration cables. At this stage of the integration, the presampler and the EMEC were near enough to connect the presampler calibration cables. While these cables are plugged on the EMEC mother-boards via low profile connectors, they are soldered to the presampler. Therefore the presampler cables were 
Table 6. Survey of both EMEC wheels in their final position inside the cryostat (vertical). All measurements concern the back rings and are given in $\mathrm{mm}$. The nominal radii are given as reference. Coordinates $(\mathrm{x}, \mathrm{y})$ of the centres of the rings are given with respect to the cryostat cold vessel axis. The accuracy is estimated to $0.3 \mathrm{~mm}$.

\begin{tabular}{|lccccc|}
\hline & Nominal & \multicolumn{2}{c}{ A wheel } & \multicolumn{2}{c|}{ C wheel } \\
& Radius & Radius & Centre & Radius & Centre \\
\hline Outer ring & 2064.0 & 2063.9 & $-0.6,-2.2$ & 2063.7 & $-0.4,-2.5$ \\
Intermediate ring & 746.0 & 746.2 & $0.0,-2.4$ & 745.6 & $0.7,-2.9$ \\
Inner ring & 398.5 & 398.1 & $-0.3,-2.1$ & 398.1 & $-0.7,-2.2$ \\
\hline
\end{tabular}

cut, stripped and soldered. A presampler TPA test was done to check the quality of the soldering.

The wheel was then inserted inside the cryostat, using the same push/pull device. During this operation, the force applied to the wheel feet was monitored. The highest recorded value was about 1 ton, while the weight of the wheel is about 26 tons. The wheel was pushed until to be in contact with the stops bolted inside the cryostat. The position of these stops defines the position of the electromagnetic calorimeter.

The cable routing on the back side was then completed, the extra lengths being distributed on the edge of the calorimeter between the front and back rings. Again a special care was taken to arrange the cables inside a layer, as uniform as possible in terms of material, inside the calorimeter envelop. High voltage cables were also routed and their ATI connectors were then mounted in the patch panels located near the top of the cryostat as described in the reference [20].

Later, the harnesses were routed in trays installed along the cold vessel wall (see figure 2). In order to avoid any ground loop, every cable tray was insulated thanks to glass epoxy strips. Moreover, kapton foils were placed between the cryostat and the cables themselves. The patch panels (signal and high voltage) were fixed on the cryostat cold wall. The wheel was then electrically tested as described in the next section.

Following the cable installation, a last geometrical measurement has been done, mainly to define with accuracy the final position of the wheel inside the cryostat. As previously, the reference was the outer cold vessel axis. Each back ring (outer, intermediate and inner) has been measured. Results are summarized in table 6 . The results are similar for both wheels. The main shift in the position of the wheel is along the vertical axis (around $2 \mathrm{~mm}$ ). There are various reasons for that:

- It has been possible to adjust the position along the horizontal axis just before the insertion, and consequently, to correct a large part of the misalignment. At the opposite, the last adjustment in vertical direction was done when the wheel was in horizontal position, and so it has been less accurate.

- After the rotation, the centre of the wheel went down by $0.8 \mathrm{~mm}$ without any possibility of correction.

- The rails inside the cryostat were not perfectly parallel to the cold vessel axis. 


\subsection{Electrical tests during integration}

\subsubsection{High voltage tests}

As discussed above, all the problematic high voltage channels seen during the assembly of the wheel were investigated when the wheel was in vertical position on T6 cradle. During this step those few problems were cured, isolating the faulty electrodes and connecting them to spare HV cables. If no faulty electrode was found a suspected HV sector was divided into two or more adding the necessary number of spare cables. Then, before and after insertion of the EMEC wheel, the high voltage holding was tested at half of the liquid argon nominal voltages. This HV test was repeated after insertion of the two HEC wheels, but no repair was possible at this time.

The results of these operations after integration of the two wheels in their cryostats can be summarized as follows:

- C wheel: $32 \mathrm{HV}$ spare lines are connected to isolated electrodes (one side of an HV sector per electrode). $3 \mathrm{HV}$ spare lines are connected to groups of electrodes.

- A wheel: $40 \mathrm{HV}$ spare lines are connected to isolated electrodes. $10 \mathrm{HV}$ spare lines are connected to groups of electrodes.

\subsubsection{Signal and calibration test}

As explained in section 6.3, the TPA test allowed to check the electrical continuity of the signal and calibration lines. In particular, it checked the integrity of the mother-boards, of the signal and calibration harnesses and all connections as well. It allowed also to verify the channel mapping.

This essential test was performed as late as possible, when the cable routing was final. For example the front side of the detector was tested just before the wheel insertion into the cryostat. Then both front and back sides were tested inside the cryostat (without any possibility to act on the front side). The result of this test on the two wheels after integration into their cryostat can be summarized as follows:

- C wheel: 2 dead channels (in the front layer), 17 strange channels;

- A wheel: 0 dead channel, 12 strange channels.

The so-called strange channels are those, as described in section 6.3, for which a non expected amplitude and/or picking time value was measured. Almost half of those channels are located in an area where an HV problem has been detected (see section 6.3).

\subsubsection{Capacitance measurement}

It was found at the beginning of year 2004 [32] that a capacitance measurement of the middle sampling for all the $\phi$ at various $\eta$ could be an important input to improve the uniformity in $\phi$ of the reconstructed energy (see section 6.4). Indeed this capacitance is bound to the liquid argon gap thickness. For the A wheel, we had the possibility to proceed to this capacitance measurement when the wheel was in vertical position on T6. Unfortunately it was too late for the $\mathrm{C}$ wheel which was already inserted in its cryostat behind both HEC wheels. For the $\mathrm{C}$ wheel we know the gap capacitances for only three modules which were measured before their assembly. Figure 23 summarizes these gap capacitance measurements. 


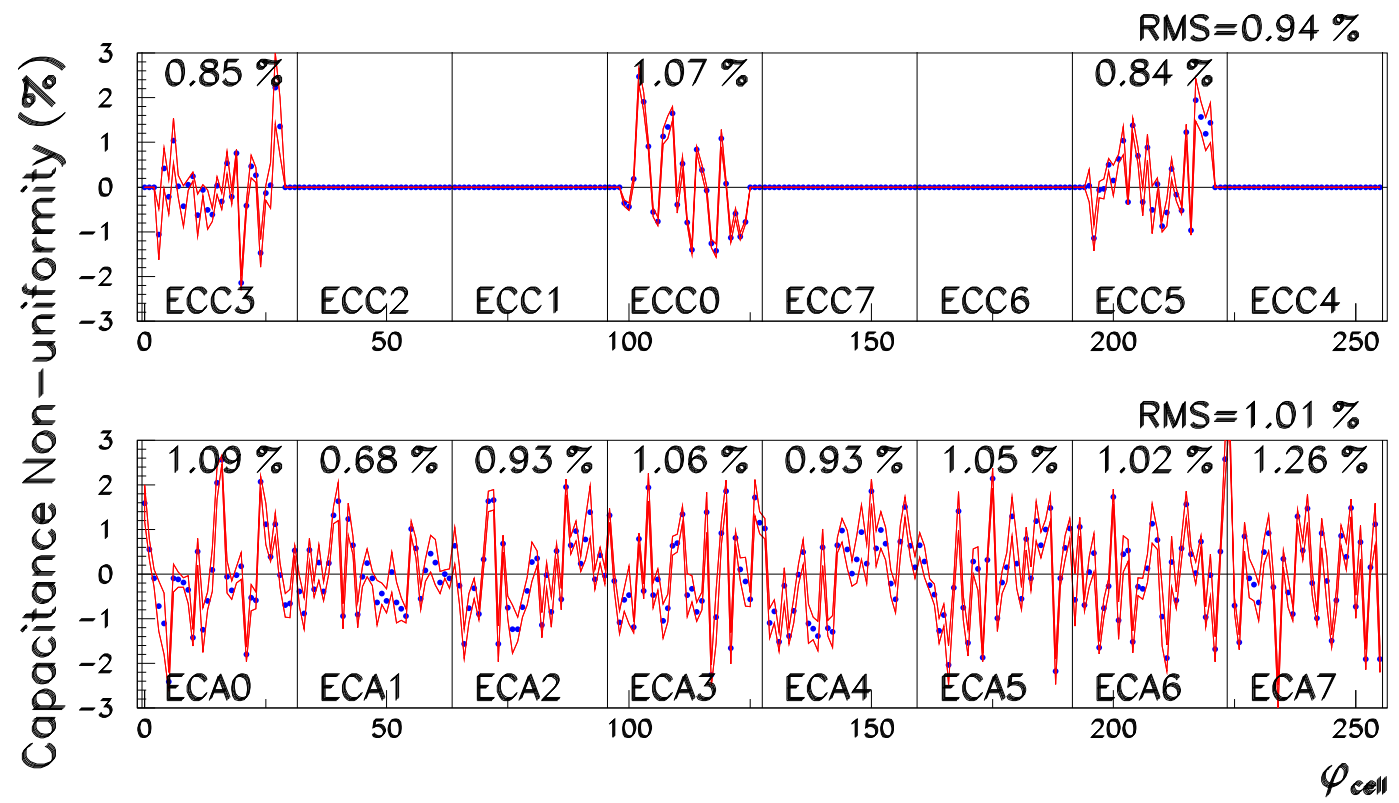

Figure 23. Measurement results of the middle cell capacitance non-uniformity along $\phi$ for the ATLAS EMEC modules. The vertical lines separate the modules. The dispersion (rms) is given for each module and for $\mathrm{A}$ and $\mathrm{C}$ wheel.

\section{Qualification tests of the calorimeter}

\subsection{Cooling down}

The cooling down process was studied on the basis of thermo-mechanical computations and of mechanical tests. First, a global analysis showed that the most critical element was the intermediate front ring, made of composite material. Thus, a very detailed analysis [33], limited to a significant portion of this ring including the ends of the corresponding bars, was carried out to study the constraints and the deformations undergone by this ring. Besides, tension and compression tests were performed on assemblies of composite pieces representative of the stresses found in the detailed analysis. This allowed to define criteria on maximal temperature differences between various points of the detector. Seven of these EMEC criteria were used to form an interlock stopping the cooling process.

Thermal models of the cryostat and of all the detectors were made to estimate a reasonable way to satisfy these criteria. To ease the homogenization of temperatures, the cryostat was filled with helium gas. The cooling down was made in two stages. From warm temperature down to $120 \mathrm{~K}$ the cooling procedure was achieved by a forced circulation cold gaseous nitrogen in the heat exchangers. Then, one circulated pressurized liquid nitrogen in the heat exchangers. The end-cap cooling lasted 50 days, at a speed of about $0.2 \mathrm{~K} / \mathrm{h}$. At $90 \mathrm{~K}$, helium was removed. The cryostat was pumped and filled with clean and dry argon gas, several times. Then the liquid argon was transferred inside the cryostat through an oxygen filter. In the ATLAS pits this operation was improved transferring liquid argon by condensation. 


\subsection{High voltage tests}

Each cryostat stayed full of liquid argon for 5 to 6 weeks. During these periods, sequences of electrical tests and measurements were performed on the detectors to qualify them. As a first test, the high voltage holding was checked. The goal was to apply the nominal HV for a rather long period: 23 (15) days for the C (A) end-cap calorimeter. Unfortunately, for the first calorimeter (ECC), because of difficulties with the stability of some HV modules, the voltage was limited to $2000 \mathrm{~V}$.

In both end-cap calorimeters about 15 short circuits have been found. In each end-cap, two of these short circuits involve both sides of a same HV sector, inducing a acceptance loss of $\Delta \eta \times \Delta \phi=0.2 \times 0.2$. A low voltage $(50 \mathrm{~V})$ was applied on all the HV lines during the cooling and filling processes. That allowed to observe that a significant fraction of the short circuits appeared when filling the cryostat with liquid argon. In order to try to cure a part of these short circuits a method was developed to burn them away. In a trial phase, this method was applied with success to some HV channels either in gazeous nitrogen (1.5 bars) or in liquid argon. This burning process has been applied later in the pit after refilling both cryostats. It has been possible to fix about $2 / 3$ of the short circuits. In most of the cases it is possible to power the short circuited HV sectors at a reduced voltage with some supplies allowing currents up to $1 \mathrm{~mA}$. Finally, as any detector region is powered at least on one side at its nominal voltage, the HV problems do not induce any dead readout cells (figure 24). They will imply some offline correction for only a small fraction $(5 \%)$ of them.

\subsection{Electrical tests}

A new TPA test, performed on each module, was repeated before and after cooling down of the cryostat to qualify each wheel before their lowering in the pit. Table 7 details the results of these tests for each signal and calibration channel. A total of 3 (4) at warm and 7(9) at cold dead channels were observed for the $\mathrm{C}(\mathrm{A})$ wheel. This is below the level of 0.5 per mil given as acceptation criterion.

Electrical measurements were also performed at warm and cold through a 64-channel multiplexing electronic board plugged on each slot of the baseplane of each feedthrough to determine:

- very precisely $(0.1 \%)$ the calibration resistance of each cell, one of the inputs for the determination of the EM scale;

- precisely (few \%) the product of the capacitance (C) and inductance (L) of each cell, to achieve a precise signal reconstruction [26];

- the general behavior of the response function and the cable attenuation with Time Domain Reflectometry (TDR) measurements on all calibration channels.

Distributions of the calibration resistances for each sampling at cold are shown in figure 25. Due to a problem during the production of these precision resistors two samples were delivered for the first sampling (S1), their mean values differing by $0.1 \%$. Any front mother-board was equipped with resistors coming from the same sample. For each sampling, the dispersion (Gaussian fit) is around $0.05 \%$, in agreement with the specifications. 

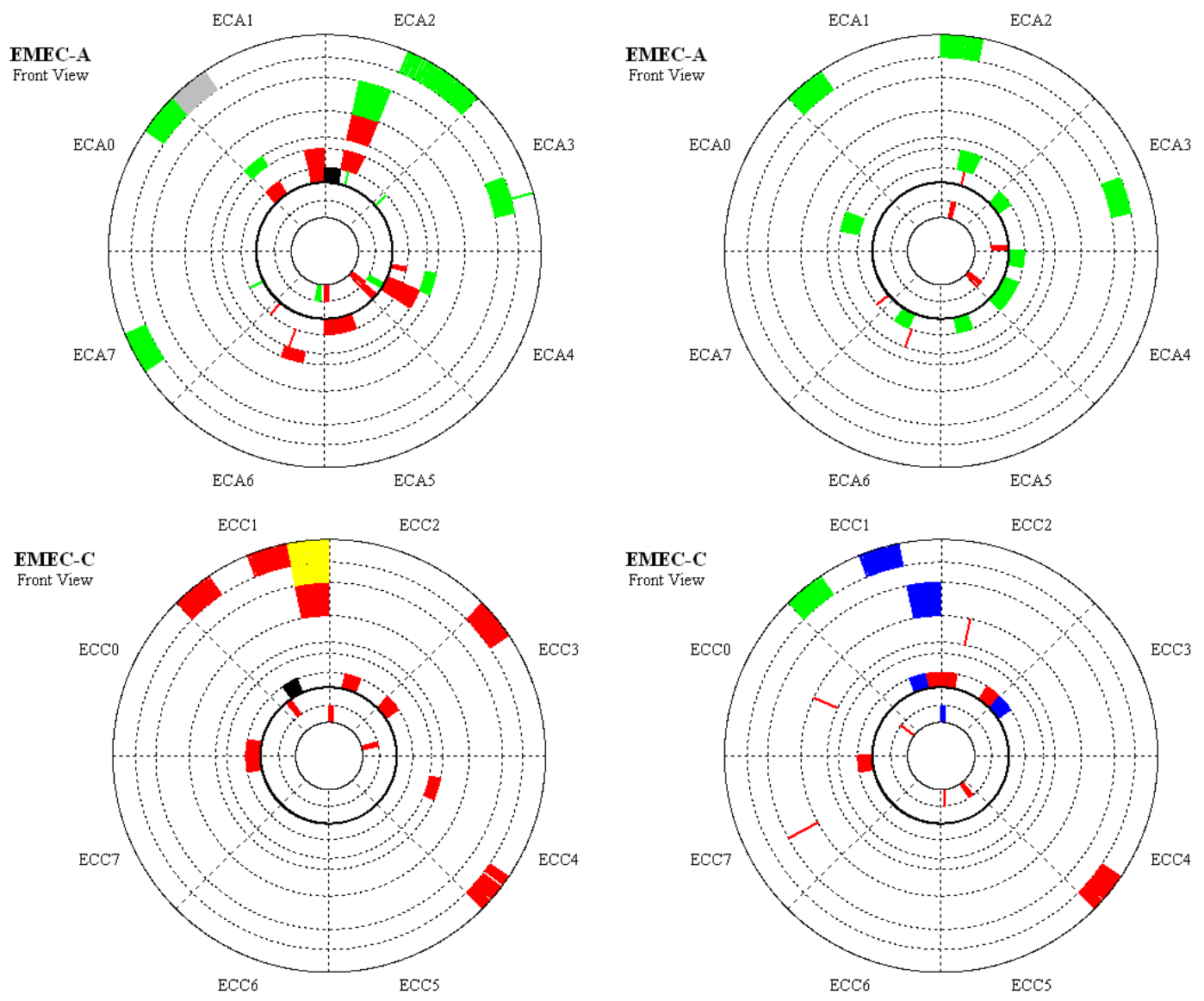

Figure 24. Mapping of the high voltage sectors with some problems after the first cold tests performed at the surface (left) and in the ATLAS pit (right). Both end-caps are seen from the interaction point. The dashed lines indicate the limits between modules and between the 7 (2) HV sectors in the outer (inner) wheel. Sectors in black were not powered on any side (short circuit on both sides). Sectors in red had a short circuit on one side only of the electrodes and were powered on the other side. Sectors in green had one side powered at lower voltage than nominal. The sector in grey of EMEC-A had one side powered at a lower than nominal voltage, while its other side was in short circuit. The two sectors of EMEC-C in yellow were in short circuit together on one side, thus powered by only one supply. Sectors of EMEC-C in blue will certainly work at a lower than nominal voltage but were not yet connected to the special power supplies.

LC measurements were performed by sending on the calibration lines a sinusoidal signal of a network analyser, with stepped $100 \mathrm{kHz}$ frequency in the range $10 \mathrm{kHz}-150 \mathrm{MHz}$. Reading the output signal allowed to determine the resonance frequency, $v_{0}=1 / \sqrt{L C}$. All middle (front) cells of outer (inner) wheel were scanned. Because of the decreasing capacitance in $\eta$, the determination of the resonance frequency is less and less precise and complicated by the variation of the signal cable length as function of $\phi$. Figure 26 shows the variation of $v_{0}$ as a function of $\eta$. A good agreement between ECA and ECC wheels is found except in the regions where $v_{0}$ is around or greater than $40 \mathrm{MHz}$ and is therefore spoiled by reflections. 
Table 7. First three columns show the total number of signal and calibration channels for the 2 wheels, the predefined acceptable maximum rate and the corresponding number of dead cells. Last two columns show the observed dead channels at warm and cold.

\begin{tabular}{|cccccc|}
\hline Layer & $\begin{array}{c}\text { Nb of } \\
\text { channels }\end{array}$ & $\begin{array}{c}\text { Max. rate } \\
\text { accepted }\end{array}$ & $\begin{array}{c}\text { Max nb } \\
\text { accepted }\end{array}$ & $\begin{array}{c}\text { Nb found } \\
\text { at warm }\end{array}$ & $\begin{array}{c}\text { Nb found } \\
\text { at cold }\end{array}$ \\
\hline Presampler & 1536 & $0.05 \%$ & 1 & 0 & 1 \\
S1 & 28544 & $2 /$ mod. & 32 & 3 & 4 \\
S2 & 23424 & $0.05 \%$ & 12 & 2 & 7 \\
S3 & 10240 & $0.05 \%$ & 5 & 0 & 2 \\
Calibration & 5952 & $0.05 \%$ & 3 & 2 & 2 \\
\hline
\end{tabular}
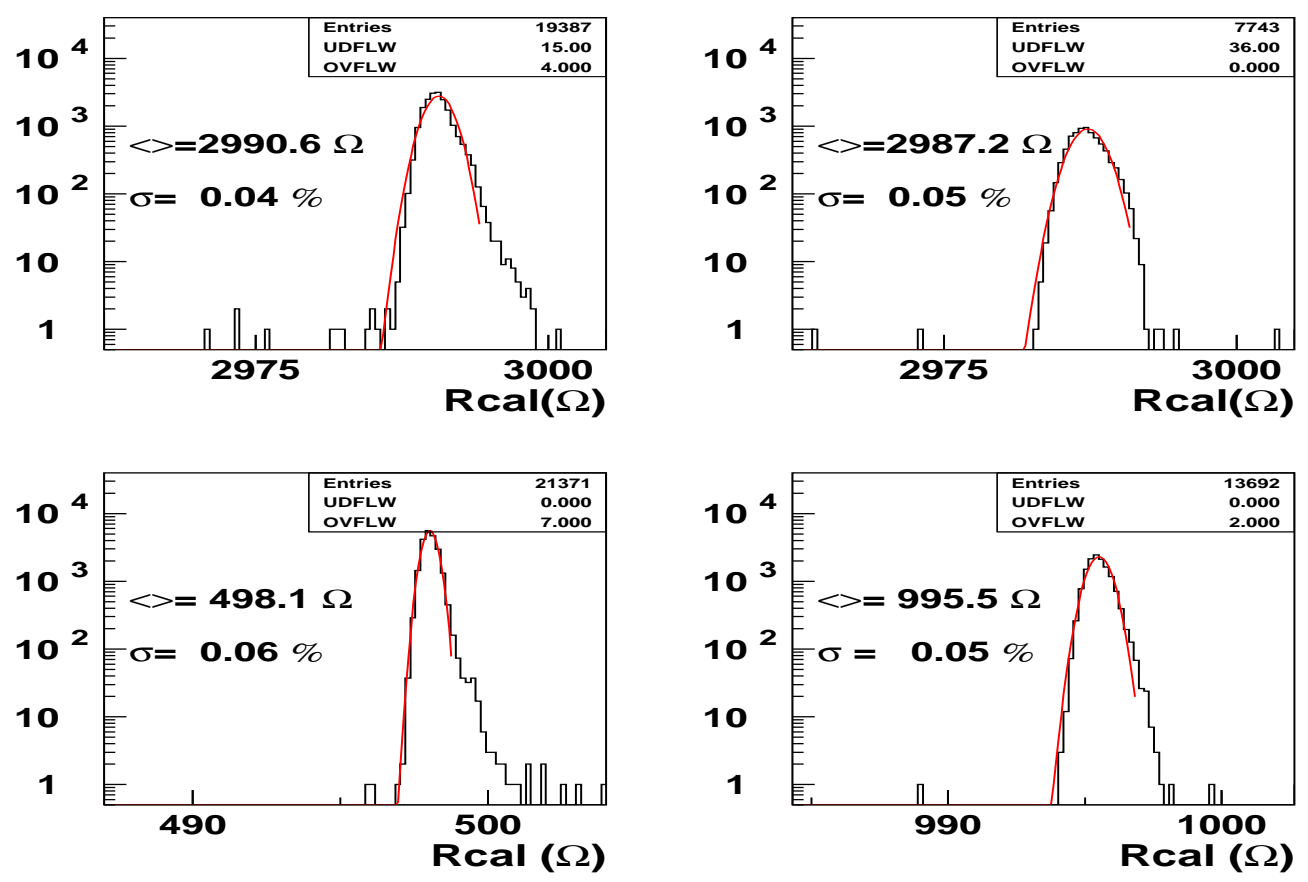

Figure 25. Resistance distributions at cold for different types of calibration injection. Mean values which should be used as input for the EM scale and dispersions are indicated.

The TDR measurements have been performed to identify possible non-uniformity, resistive discontinuity and anomalous response in the calibration lines. The qualitative analysis of the results have been used to identify problematic lines and as an independant cross check of the results of TPA tests.

\section{Conclusions}

The physics to be studied at the LHC imposes very stringent constraints on the electromagnetic 


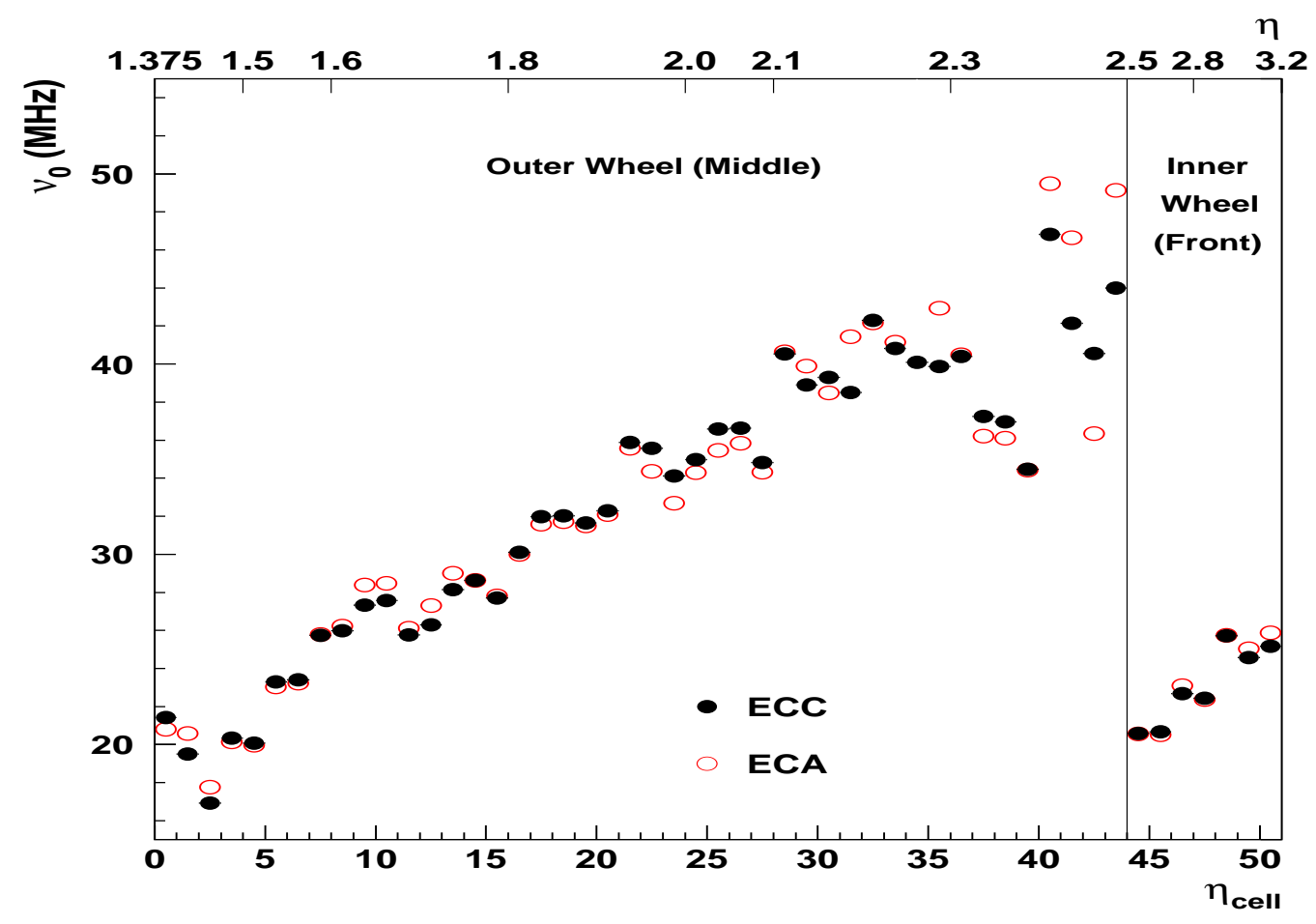

Figure 26. Resonance frequency at cold ( $v_{0}$ in $\mathrm{MHz}$ ) averaged in $\phi$ as a function of $\eta$ cell number in the middle (front) sampling of the outer (inner) wheel.

calorimetry. Furthermore the harsh environment of the LHC requires the techniques to be pushed to the limit. These considerations led ATLAS to choose a liquid argon / lead sampling calorimeter with an accordion geometry. This choice implies a variation of the absorber-electrode gap with the pseudorapidity, hence a very complex geometry. The construction of the end-cap calorimeters has taken eight years from the first designs to the finished detector. All along the construction, from component construction, module assembly to wheel insertion inside the cryostat, stringent quality assurance steps were taken to minimize detector imperfections. The same care was brought to the construction of the cryostats and associated feedthroughs. At the end of the construction phase, i.e. after the cold test of each wheel, the number of defects, such as dead signal channels or faulty HV sectors, is well below the predefined acceptation criteria. Three out of 16 series end-cap modules have been calibrated with electrons. The results obtained on the uniformity response, the energy and position measurements meet the challenging physics requirements despite the complex geometry of these end-caps.

The end-cap calorimeters are now in place and cold in the ATLAS pit. The next ongoing important step is their commissioning.

\section{Acknowledgments}

We are indebted to our technicians for their excellent contribution to the construction and the running of EMEC modules, of the end-cap cryostats, of the feedthroughs and of the associated electronics. 
We would like to thank F. Gallardo from UAM (Madrid), J. Balsategui from Talleres Aratz (Madrid), F. Léon, M. Mathieu, P. Ollive, C. Olivier, D. Tézier, F. Peillon, F. Rivière from CPPM (Marseille), F. Alberti, A. Andreani, B. Monticelli, F. Sabatini from Milano, R.G.Snopkov from BINP (Novosibirsk), J. Bonenfant, J. Bonis, C. Bourgeois, P. Favre, M. Quentin, A. Thiebault, P. Imbert from LAL (Orsay), Ph. Beauchet, A. Commercon, G. Descote, ${ }^{23} \mathrm{Ph}$. Laloux, D. Laporte, Ph. Repain, D. Steyaert, Y. Tréguier from LPNHE (Paris).

The construction of the electromagnetic end-cap calorimeters would not have been possible without the important financial support of our funding agencies. This support is kindly acknowledged.

\section{References}

[1] ATLAS collaboration, Calorimeter Performance Technical Design Report, CERN/LHCC/96-040 (1996), http://atlasinfo.cern.ch/Atlas/TDR/caloperf/caloperf.html.

[2] ATLAS collaboration, ATLAS: Detector and Physics Performance Technical Design Report, Volumes I and II, CERN-LHCC-99-014 CERN-LHCC-99-015 (1999), http://atlas.web.cern.ch/Atlas/GROUPS/PHYSICS/TDR/access.htm1.

[3] RD3 collaboration, B. Aubert et al., Performance of a liquid argon electromagnetic calorimeter with an accordion geometry, Nucl. Instrum. Meth. A 309 (1991) 438.

[4] B. Aubert et al., Performance of a liquid argon Accordion calorimeter with fast readout, Nucl. Instrum. Meth. A 321 (1992) 467.

[5] RD3 collaboration, D.M. Gingrich et al., Performance of an endcap prototype of the ATLAS accordion electromagnetic calorimeter, Nucl. Instrum. Meth. A 389 (1997) 398.

[6] ATLAS collaboration, Technical Proposal for a General-Purpose pp Experiment at the Large Hadron Collider at CERN, CERN/LHCC/94-043 (1994).

[7] ATLAS collaboration, ATLAS Liquid Argon Calorimeter Technical Design Report, CERN/LHCC/96-041 (1996), http://atlas.web.cern.ch/Atlas/GROUPS/LIQARGEXT/TDR/Welcome.htm1.

[8] ATLAS Electromagnetic Barrel Liquid Argon Calorimeter Group, B. Aubert et al., Construction, assembly and tests of the ATLAS electromagnetic barrel calorimeter, Nucl. Instrum. Meth. A 558 (2006) 388.

[9] ATLAS Electromagnetic Liquid Argon Calorimeter Group, B. Aubert et al., Performance of the ATLAS electromagnetic calorimeter end-cap module 0, Nucl. Instrum. Meth. A 500 (2003) 178.

[10] B. Aubert et al., Development and construction of large size signal electrodes for the ATLAS electromagnetic calorimeter, Nucl. Instrum. Meth. A 539 (2005) 558.

[11] J. Colas et al., Position resolution and particule identification with the ATLAS EM calorimeter, Nucl. Instrum. Meth. A 550 (2005) 96.

[12] V. Aulchenko et al., Performance of the EM end-cap Presampler in the 1999 Test Beam Run, ATLAS Note ATL-LARG-2001-016(2001).

[13] G. García et al., Automatic thickness control system for the ATLAS electromagnetic calorimeter absorber plates, Nucl. Instrum. Meth. A 418 (1998) 513.

\footnotetext{
${ }^{23}$ deceased
} 
[14] J. Collot et al., A neutron irradiation facility featuring cryogenic temperatures and dedicated to Large Hadron Collider detector design, Nucl. Instrum. Meth. A 350 (1994) 525.

[15] W. Bonivento and D. Lacour, Acceptable Values of Resistances on Electrodes of the ATLAS em Calorimeter, ATLAS Note ATL-LARG-1999-019 (1999).

[16] P. Barrillon et al., Electrical tests for the validation of the electromagnetic end-cap calorimeter modules, ATLAS Note ATL-LARG-2003-004 (2003).

[17] F. Berny et al., A protection scheme for the calibration resitive networks, ATLAS Note ATL-LARG-2003-005 (2003).

[18] P. Pralavorio and D. Sauvage, Review of the crosstalk in the module 0 of the Electromagnetic Endcap Calorimeter, ATLAS note ATL-LARG-2001-006 (2001).

[19] F. Hubaut et al., Crosstalk in production modules of the Electromagnetic Endcap Calorimeter, ATLAS note ATL-LARG-2003-012 (2003).

[20] C. Cerna, Description of the HV channels for the EMEC A and C wheels, ATLAS Note EDMS ATL-AE-AN-0003 (2003).

[21] S. Filimonov and P. Puzo, Temperature readout for the Liquid Argon End-Cap Calorimeters, ATLAS Note EDMS ATL-AP-IN-0002 (2004) and references inside.

[22] M. Adams et al., A purity monitoring system for liquid argon calorimeters, Nucl. Instrum. Meth. A 545 (2005) 613.

[23] NA31 collaboration, H. Burkhardt et al., The beam and detector for a high-precision measurement of the CP violation in neutral-kaon decays, Nucl. Instrum. Meth. A 268 (1988) 116.

[24] J. Bremer et al., Cryogenic System for the Test Facilities of the ATLAS Liquid Argon Calorimeter Modules, CERN-LHC-Project-Report-234.

[25] J. Spanggaard, Delay Wire Chambers - A Users Guide, SL-Note-98-023, http://sl.web.cern.ch/SL/Publications/bi98-023.pdf.

[26] M. Aharrouche et al., Response Uniformity of the ATLAS liquid argon electromagnetic calorimeter, Nucl. Instrum. Meth. A 582 (2007) 429.

[27] G. Macé, S. Prat and J.J. Veillet, Description and construction of the cryostats of the Atlas end cap liquid argon calorimeters, LAL/RT 06-04 (2006), http://publication.lal.in2p3.fr/2006/LALRT0604.pdf.

[28] D.M. Gingrich et al., Construction, Assembly and Testing of the ATLAS Hadronic End-cap Calorimeter, 2007 JINST 2 P05005.

[29] A. Artamonov et al., The ATLAS Forward Calorimeters, 2008 JINST 3 P02010.

[30] D. Axen et al., Signal feedthroughs for the ATLAS barrel and endcap calorimeters, Rev. Sci. Instrum. 76 (2005) 063306.

[31] B. Botchev et al., The High Voltage Feedthroughs for the ATLAS Liquid Argon Calorimeters, 2007 JINST 2 T10002.

[32] F. Hubaut and C. Serfon, Response uniformity of the ATLAS electromagnetic endcap calorimeter, ATLAS Note ATL-LARG-2004-015 (2004)

[33] P. Karst, Specification for the cooling and heating of the EMEC, ATLAS Note EDMS ATL-AE-ES-0020 (2004). 